\title{
Evaluation quality of service for internet of things based on fuzzy logic: a smart home case study
}

\author{
Lairedj Aboubaker Saddik ${ }^{1}$, Ben Ahmed Khalifa ${ }^{1}$, Bounaama Fateh ${ }^{2}$ \\ ${ }^{1}$ Department of Mathematics and Computer Science, Faculty of Exact Sciences, Tahri Mohammed University, Bechar, Algeria \\ ${ }^{2}$ Department of Electrical Engineering, Faculty of Technology, Tahri Mohammed University, Bechar, Algeria
}

\begin{tabular}{l} 
Article Info \\
\hline Article history: \\
Received May 31,2021 \\
Revised Dec 20, 2021 \\
Accepted Dec 29, 2021 \\
\hline Keywords: \\
Fuzzy logic \\
Fuzzy performance importance \\
Index \\
Fuzzy quality of service index \\
Internet of thing \\
Sustainability quality of service
\end{tabular}

\begin{abstract}
The development of the internet of thing (IoT) technology has become a major concern in sustainability of quality of service (SQoS) in terms of efficiency, measurement, and evaluation of services, such as our smart home case study. Based on several ambiguous linguistic and standard criteria, this article deals with quality of service (QoS). We used fuzzy logic to select the most appropriate and efficient services. For this reason, we have introduced a new paradigmatic approach to assess QoS. In this regard, to measure SQoS, linguistic terms were collected for identification of ambiguous criteria. This paper collects the results of other work to compare the traditional assessment methods and techniques in IoT. It has been proven that the comparison that traditional valuation methods and techniques could not effectively deal with these metrics. Therefore, fuzzy logic is a worthy method to provide a good measure of QoS with ambiguous linguistic and criteria. The proposed model addresses with constantly being improved, all the main axes of the QoS for a smart home. The results obtained also indicate that the model with its fuzzy performance importance index (FPII) has efficiently evaluate the multiple services of SQoS.
\end{abstract}

This is an open access article under the CC BY-SA license.

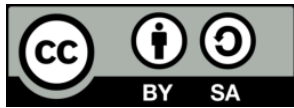

\section{Corresponding Author:}

Lairedj Aboubaker Saddik

Department of Mathematics and Computer Science, Faculty of Exact Sciences

Tahri Mohammed University

Bechar, Algeria

Email: lairedjboubakerdz@gmail.com

\section{INTRODUCTION}

Internet of things (IoT) technology is characterized by several challenges. Including technical challenges such as battery capacity, storage, energy and others. Efficiency challenges such as the quality of communication between devices, and sustainability of services. in addition, the difficulty in developing a framework to achieve a better evaluation of the quality of service (QoS) [1], which could be among the most important challenges for researchers [2]. Establishing precise measurement criteria is a complex issue, because it is related to an immaterial aspect and a heterogeneous level of QoS [3]. It is difficult to determine the limitations of clear service activities, including a variety of different IoT network situations. Clients and objects who obtaining the same service or task could have very different perceptions of QoS classification [4]. Different criteria in various situations lead to not guaranteeing the same satisfaction in ensuring QoS. For example, the real response time in an IoT system for industrial control has completely different requirements and criteria from those of smart city or smart healthcare [5]. The ability to maintain a consistent level of QoS for different customers is essential across all networks [4], [5]. According to these challenges, the main problem is how to develop a general mechanism to evaluate all the services of any IoT system. Therefore, we 
have presented a smart home case study, because they are the closest to our daily lives and have a great deal of importance in an individual's life, especially in the Corona virus.

Management of QoS becomes more difficult when execute it in IoT. In addition, there is a need to establish a comprehensive assessment framework for QoS, that meets all requirements to ensure sustainability of quality of service (SQoS), and ensures the robustness of the IoT system. Moreover, several studies have been devoted to the development of Qo based on fuzzy logic system. For example, Jiang et al. [1] proposed a novel QoS optimization paradigm for the IoT systems based on fuzzy logic and visual information in the field of the IoT industry. They proposed a correlation algorithm to optimize the IoT environment using a fuzzy system and visual information mining technology. Furthermore, Wibowo and Grandhi [6] presented a fuzzy multi-criteria analysis model for evaluating the performance of IoT-based supply chains. By using technique similarity to the ideal solution (TOPSIS) approach, for determining the overall performance of each alternative. This proposition aims to evaluate the performance of IoT-based supply chains for improving their competitiveness. Wibowo [7] provided a definition of different measures of QoS related to the components of IoT applications. Also, they were touch on the issue of human decisionmaking and the placement of risk in these decisions. An algorithm was developed to produce a performance index for every supply chain, based on IoT alternatives across all selection criteria. Li et al. [8] presented a dynamic QoS solution based on the differentiated services (DiffServ) approach for enterprise in a real network, upon a fuzzy interpolation approach with a TSK style rule base. Kazmi et al. [9] proposed an approach for maximizing a packet delivery ratio of the IoT network, by implementing fuzzy logic-based link quality estimators in the WiseRoute. Araújo et al. [10] proposed an approach for IoT route selection by implementing the fuzzy logic in order to attain the requirements of specific applications. Choosing dynamically of information for objective functions of the routing protocol for low power and lossy networks (RPL). Sankar and Srinivasan [11] proposed a fuzzy logic-based energy aware routing protocol (FLEARPL). Which considers some metrics like residual energy (RER), and an expected transmission count (ETX). Its objective is to select the best route to transfer the network data efficiently. This study is evaluated by comparison with similar protocol standard RPL, MRHOF (ETX) based RPL (MRHOF-RPL), and FL-RPL. Retima et al. [12], a fuzzy logic-based framework is proposed, which by QoS evaluated within distributed context manager and context-aware applications. In addition, it used MapReduce for quicker computing and parallelism processing. Another study was performed by [13], to improve the lifespan of IoT network. Where they proposed an energy-saving mechanism based on fuzzy logic. They have improved learning compared to IEEE 802.15.4 protocol.

From this review, we can see many researches providing some insights into different aspects of QoS using fuzzy logic. Therefore, the fuzzy logic system has become important in several fields of scientific research. As it is able to manage fuzzy information, and efficiently handle complex modeling [14]. In addition, fuzzy logic has stronger semantics than a set of field methodology like machine-learning, reinforcement learning, because of its intuitive expressive and the powerful reasoning ability [15]. Thus, the most important point is to understand the SQoS criteria, to analyzing, integrate, and convert into strategic measuring capabilities. On this basis, The authors found that no special research for evaluation all requirements of QoS. For this reason, the authors presented an advanced model for assessing the sustainable QoS of the internet of things, based on fuzzy logic, which it was a research study for smart homes. Furthermore, a sustainable QoS makes the same satisfaction to the changing criteria for diversity of IoT systems [6], [16], [17].

Based on a comprehensive survey of a smart home research review by the authors, they have collected an appropriate information in terms of language variables [17], [18]. This model is based on a general study of all indicators and linguistic variables of criteria/metrics. The model has two important steps. Firstly, verifying QoS inputs at the smart home level by matching the input values (fuzzy quality of service indices (FQSI)), with the marks of the criteria of QoS. Secondly, identifying the obstacles (or weakest services) to improve QoS performance by calculating the fuzzy performance importance index (FPII).

The rest of the paper presented as follows: in section 2 offer a presentation of description of our approach. Section 3 exhibits the simulation results of our proposed techniques and eventually. Section 4 concludes our paper and perspectives.

\section{METHOD}

The scope of this study is on several factors. Establishing a QoS sustainability mechanism. Addressing all solutions to challenges as mentioned in the previous paragraph. A conceptual model for assessing QoS based on fuzzy logic is developed. Where it is contains three basic levels. Firstly, identification of information and QoS criteria, by collecting and analyzing the development of IoT. Secondly, assessing QoS capabilities to obtain QoS for sustainable internet of things based fuzzy. Thirdly, 
determination an FPII score to specify the weakest areas for improving QoS performance [6]. The process takes place in four steps, as shown in Figure 1.

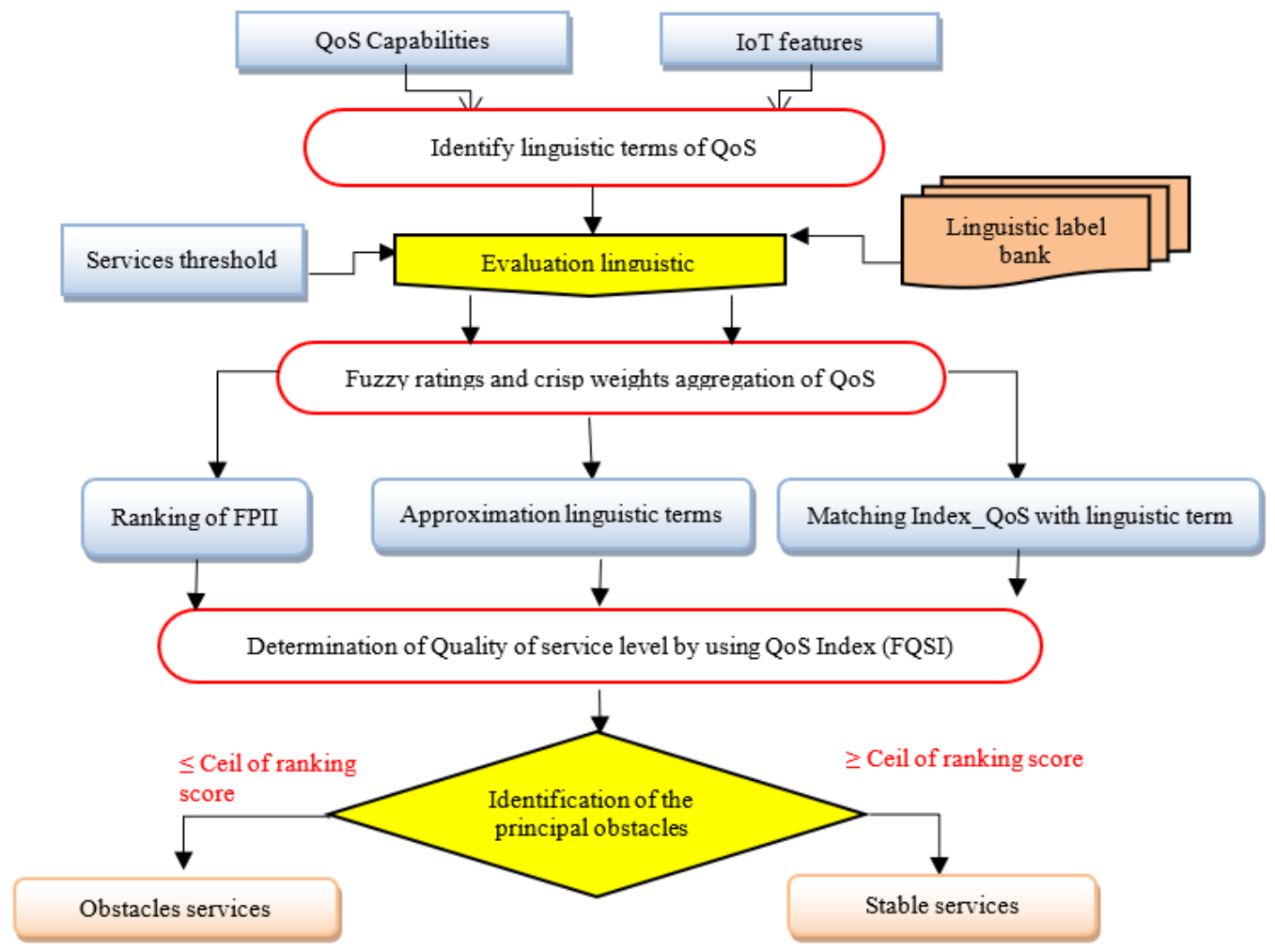

Figure 1. Framework steps to measure and evaluate the QoS for IoT

The description steps are represented as follows:

- $\quad$ Selection criteria, designations and attributes for assessing service quality.

- $\quad$ Selecting the appropriate language for QoS attributes

- $\quad$ Measuring performance ratings and importance weights for QoS

- $\quad$ Rounding linguistic terms from ambiguous numbers

- $\quad$ Determine FQSI using fuzzy weights

- $\quad$ Determining the Euclidean distance to match the FQSI with the approximate QoS level

- Matching FQSI.

- $\quad$ Analyze the main obstacles.

In this work, we proposed three basic algorithms: The criteria selection algorithm, which is for the identification of information and criteria of QoS by collecting and analyzing the IoT development. The defining a fuzzy quality of service index (FQSI) algorithm, which is to evaluate QoS capabilities and synthesize the ratings $\mathrm{Ri}$ and the weights $\mathrm{Wi}$ to obtain an FQSI. The fuzzy performance importance index (FPII) algorithm, which is determining the FPII to compute and identify weaker services to offer proposals for improvement of QoS performance. The following Table 1 represents the set of used notations in our paper.

Table 1. Notaion table

\begin{tabular}{cc}
\hline Notation & Description \\
\hline FQSI & Fuzzy Quality of service index \\
FPII & Fuzzy performance importance index \\
QSI & Quality of services index \\
QS & Is the natural language expression \\
R & Performance rating \\
W & Importance weighting \\
ED & Euclidean Distance \\
a, b, c & The numbers for fuzzy triangular: Low, middle, and upper numbers \\
Rs & Ranking score \\
\hline
\end{tabular}




\subsection{Criteria selection algorithm}

The first step depends on the selection of attributes and criteria related to the evaluation of the QoS criteria. Determine the appropriate linguistics terms for QoS attributes through systematic study and literature review. We proposed three levels: QoS layers, service standards, and service attributes. From experts and decision-makers, are collected and measured the performance ratings (R), the linguistic terms, and importance weights. Then, the linguistic terms are approximated to ambiguous numbers by fuzzy logic [10]. Which is a tool for converting human knowledge and their ability to make decisions into a mathematical formula. Using a fuzzy process [17], [18], the quality of service index (QSI) is constructed and determined by:

$$
\begin{aligned}
& Q S I_{i}=\sum_{j=1}^{N} Q S_{i j} \\
& \mathrm{QSI}=\sum_{i=1}^{N} R_{i} \times W_{i}
\end{aligned}
$$

where, $Q S_{i j}$ is the QoS levels of capability $\mathrm{j}$ of system $\mathrm{i}$, and $R_{i j}$ and denote the SQoS index, and $W_{i j}$ The weight of each SQoS capability.

$$
\sum_{j=1}^{N} W_{i}=1
$$

The fuzzy index of the SQoS criteria of level two can be calculated as:

$$
Q S I_{i j}=\frac{\sum_{k=1}^{N}\left(Q S_{i j k} \times W_{i j k}\right)}{\sum_{i=1}^{N} W_{i j k}}
$$

where $Q S_{i j k}$ represents performance $\left(R_{i j k}\right)$ rating and $W_{i j k}$ Represents a weight importance of QoS attributes [18]. The following Algorithm 1 shows the methodology of the first phase of this proposed model:

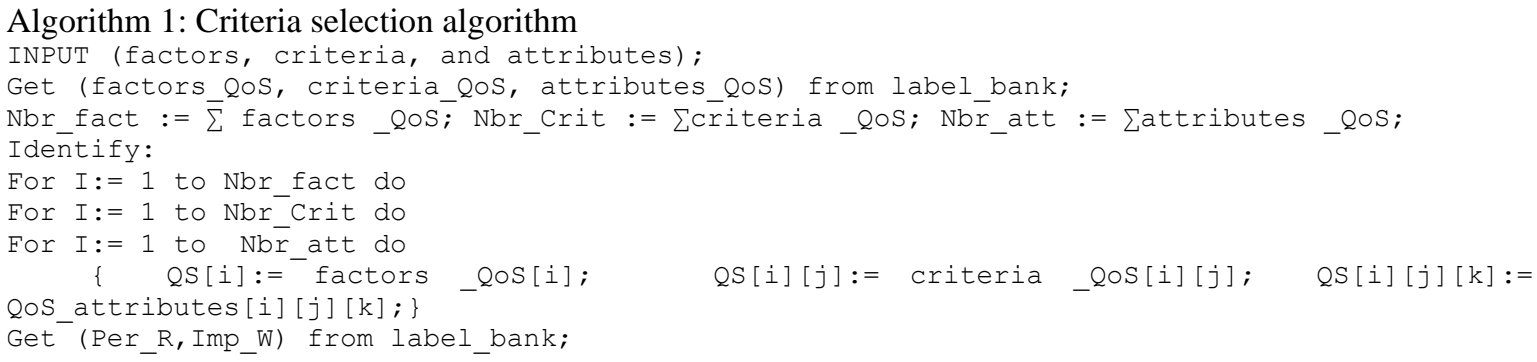

\subsection{Defining a fuzzy quality of service index algorithm}

Synthesizing Ri scores and Wi weights as follows:

$$
\begin{aligned}
& R_{i j}=\frac{\sum_{k=1}^{N}\left(R_{i j k} \times W_{i j k}\right)}{\sum_{i=1}^{N} W_{i j k}} \\
& R_{i}=\frac{\sum_{j=1}^{N}\left(R_{i j}\right)}{\sum_{j=1}^{N} j}
\end{aligned}
$$

by using, the (5) it can be calculated FQSI:

$$
F Q S I=\frac{Q S I}{\sum_{i=1}^{N} W_{i}}
$$

to match this latter FQSI value with a natural expression. We use the Euclidean distance method [18] to find the approximate QoS level. By calculating, the distance between given fuzzy number and each of fuzzy numbers representing a set of natural language expressions. It can be calculated as follows:

$$
D(Q S I, Q S)=\left\{\sum_{x \in p},\left[f_{Q S I}(x)-f_{Q S}(x)\right]^{2}\right\}^{1 / 2}
$$

where QS is the natural language expression. The following Algorithm 2 shows the steps of the second phase. 
Algorithm 2: Defining a fuzzy quality of service index (FQSI)

INPUT (services threshold);

If services thrēshold is true then Evaluate linguitstic:

Set (Imp_W \& Per_R) in QS;

Return (table $Q \bar{S})$;

Calcule (QSI [i], QS [i] [j]);

set (R) in table $Q S$;

Calcule (FQSI);

if (R) is true then get (QSI_level) from label_bank; Return QSI_level;

Distanceecludien (FQSI, QSI_level); Matchng (FQSI, D) ;

\subsection{Determining the fuzzy performance importance index algorithm (FPII)}

Determining the fuzzy performance importance index algorithm as shown in Algorithm 3. FPII is used to identify obstacles and identify weaker services [16]-[19]. FPII is calculated as follows:

$$
F P I I_{i j k}=W_{i j k}^{\prime} \times Q S_{i j k}
$$

where:

$$
\left.W_{i j k}^{\prime}=\left[\begin{array}{lll}
1 & 1 & 1
\end{array}\right)-W_{i j k}\right]
$$

$W_{i j k}$ Is the fuzzy importance weight of the QoS for IoT element capability ijk.

Since fuzzy numbers do not always give a totally ordered set as do real numbers [17], [20]. All FPIIs must be classified. Here there are many measures of similarity between fuzzy numbers [20]. In order to be identified the main obstacles, we rely on the fuzzy number ranking of the centroid method [18], [20] membership function (a, b, c) as shown in a (10), where a, b, c, is the lower, medium, and upper triangular fuzzy numbers, defined as:

$$
\text { Ranking Score }=(a+4 b+c) / 6
$$

Algorithm 3: Determining the fuzzy performance importance index (FPII)

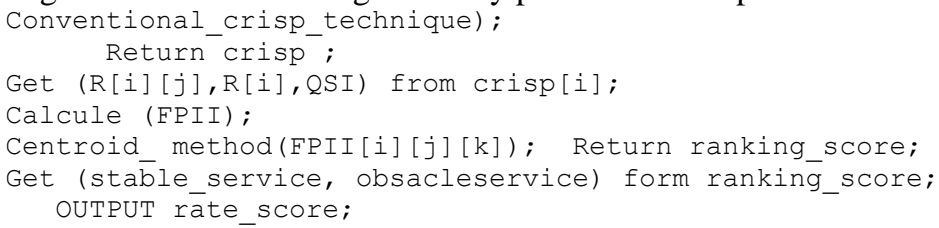

\section{RESULTS AND DISCUSSION}

In this section, we developed the conceptual model for sustainability QoS assessment. Where the analysis study carried out based on measuring the presented parameters was conducted for a smart home based on the IoT system. The only difference is in the collection of data that may differ in diverse cases. The Matlab simulator was used to validate this proposed approach [21].

\subsection{Selection of criteria, labels, and attributes}

To assess QoS, we used a combination of advanced information technologies, and service management technology of smart homes. The model has been developed from literature analysis [22]-[31], and through experts. As a result, as shown in Table 2, there are three levels: QoS layer, QoS Criteria, QoS services.

\subsection{Determining appropriate language terms for QoS attributes}

In order to evaluate the performance of the QoS capabilities. Therefore, the linguistic terms in this approach are used to assess the performance rating and importance weights of the QoS capabilities, based on the original data of the study in [18], as shown in Table 3. They are as follows: excellent (E), very good $(\mathrm{VG})$, good $(\mathrm{G})$, fair $(\mathrm{F})$, poor $(\mathrm{P})$, very poor $(\mathrm{VP})$, and worst $(\mathrm{W})\}$ these terms are selected to assess the performance rating of QoS capabilities. In relation to the importance of QoS weights attributes, and very high $(\mathrm{VH})$, high $(\mathrm{H})$, fairly high $(\mathrm{FH})$, medium $(\mathrm{M})$, fairly low $(\mathrm{FL})$, low (L), very low (VL) in terms of assessing the performance of QoS capabilities. 
Table 2. Define the appropriate linguistic terminology for QoS attributes [22]-[31]

\begin{tabular}{|c|c|c|}
\hline QoS Layer Index (QSi) & QoS Criteria Index (QSij) & QoS services Index (QSijk) \\
\hline \multirow{10}{*}{$\begin{array}{l}\text { Application services } \\
\text { layer }\left(\mathrm{QS}_{1}\right)\end{array}$} & Smart Homes design & Design of data collection procedures $\left(\mathrm{QS}_{111}\right)$ \\
\hline & Interface $\left(\mathrm{QS}_{11}\right)$ & Authorization and Authentication design $\left(\mathrm{QS}_{112}\right)$ \\
\hline & & Design of technological infrastructure $\left(\mathrm{QS}_{113}\right)$ \\
\hline & Remote devices services & External IoT house-devices $\left(\mathrm{QS}_{121}\right)$ \\
\hline & $\left(\mathrm{QS}_{12}\right)$ & Stationary IoT house-devices $\left(\mathrm{QS}_{122}\right)$ \\
\hline & & Supportive IoT house-devices $\left(\mathrm{QS}_{123}\right)$ \\
\hline & Applications technologies & Information and communication technology $\left(\mathrm{QS}_{131}\right)$ \\
\hline & $\left(\mathrm{QS}_{13}\right)$ & Telemetry: technologies to exchange information $\left(\mathrm{QS}_{132}\right)$ \\
\hline & & Systems strategies and new technologies surveillance $\left(\mathrm{QS}_{133}\right)$ \\
\hline & & Mobile operator assistants $\left(\mathrm{QS}_{134}\right)$ \\
\hline \multirow{15}{*}{$\begin{array}{l}\text { Remote services layer } \\
\left(\mathrm{QS}_{2}\right)\end{array}$} & Information Processing & Information accuracy $\left(\mathrm{QS}_{211}\right)$ \\
\hline & $\begin{array}{l}\text { Design: derines the robust } \\
\text { and accurate information } \\
\text { capturing }\left(\mathrm{QS}_{21}\right)\end{array}$ & $\begin{array}{l}\text { Trustworthiness }\left(\mathrm{QS}_{212}\right) \\
\text { provenance of IoT }\left(\mathrm{QS}_{213}\right)\end{array}$ \\
\hline & Remote control of house & House Items $\left(\mathrm{QS}_{221}\right)$ \\
\hline & system $\left(\mathrm{QS}_{22}\right)$ & Remote IoT alarm $\left(\mathrm{QS}_{222}\right)$ \\
\hline & & Virtual monitoring of important devices $\left(\mathrm{QS}_{223}\right)$ \\
\hline & & Remote Emergency $\left(\mathrm{QS}_{224}\right)$ \\
\hline & & Remote display and operation $\left(\mathrm{QS}_{225}\right)$ \\
\hline & Improve system & Identification systems $\left(\mathrm{QS}_{231}\right)$ \\
\hline & performance $\left(\mathrm{QS}_{23}\right)$ & Interconnected rooms information system $\left(\mathrm{QS}_{232}\right)$ \\
\hline & & House System diversity $\left(\mathrm{QS}_{233}\right)$ \\
\hline & & Accessibility systems $\left(\mathrm{QS}_{234}\right)$ \\
\hline & & Operations and workflow management $\left(\mathrm{QS}_{235}\right)$ \\
\hline & Computing & Web server $\left(Q_{241}\right)$ \\
\hline & Technologies $\left(\mathrm{QS}_{24}\right)$ & Cloud computing, edge computing, fog computing $\left(\mathrm{QS}_{242}\right)$ \\
\hline & & Nodes: identify central, cluster nodes and a base station $\left(\mathrm{QS}_{243}\right)$ \\
\hline \multirow{8}{*}{$\begin{array}{l}\text { Knowledge services layer } \\
\left(\mathrm{QS}_{3}\right)\end{array}$} & Human operator $\left(\mathrm{QS}_{31}\right)$ & Human trust autonomous systems $\left(\mathrm{QS}_{311}\right)$ \\
\hline & & Monitoring for a house with Presence of people $\left(\mathrm{QS}_{312}\right)$ \\
\hline & & Improved house management $\left(\mathrm{QS}_{313}\right)$ \\
\hline & Disease management & Improved diagnostic and treatment $\left(\mathrm{QS}_{321}\right)$ \\
\hline & $\left(\mathrm{QS}_{32}\right)$ & Decreased cost $\left(\mathrm{QS}_{322}\right)$ \\
\hline & & Enhanced human management $\left(\mathrm{QS}_{323}\right)$ \\
\hline & Decision-making systems & Traceability of all information $\left(\mathrm{QS}_{331}\right)$ \\
\hline & $\left(\mathrm{QS}_{33}\right)$ & The machine understanding, semantic technology $\left(\mathrm{QS}_{332}\right)$ \\
\hline \multirow{14}{*}{$\begin{array}{l}\text { Sensing services layer } \\
\left(\mathrm{QS}_{4}\right)\end{array}$} & Sensor IoT $\left(\mathrm{QS}_{41}\right)$ & Technology things appropriate sensor to collect data $\left(\mathrm{OS}_{411}\right)$ \\
\hline & & Power consumption rate $\left(\mathrm{QS}_{412}\right)$ \\
\hline & & Sensing technologies $\left(\mathrm{QS}_{413}\right)$ \\
\hline & Data acquisition $\left(\mathrm{QS}_{42}\right)$ & Data structure $\left(\mathrm{QS}_{421}\right)$ \\
\hline & & On-time access $\left(\mathrm{QS}_{422}\right)$ \\
\hline & & Data sources $\left(\mathrm{QS}_{423}\right)$ \\
\hline & & Data integration platforms $\left(\mathrm{QS}_{424}\right)$ \\
\hline & & Data accuracy $\left(\mathrm{QS}_{425}\right)$ \\
\hline & & Storage capacity. $\left(\mathrm{QS}_{426}\right)$ \\
\hline & & Critical data $\left(\mathrm{QS}_{427}\right)$ \\
\hline & Reliability and & Improve endurance $\left(\mathrm{QS}_{431}\right)$ \\
\hline & Continuity $\left(\mathrm{QS}_{43}\right)$ & Resource efficiency $\left(\mathrm{QS}_{432}\right)$ \\
\hline & & Complexity $\left(\mathrm{QS}_{433}\right)$ \\
\hline & & Scalability $\left(\mathrm{QS}_{434}\right)$ \\
\hline \multirow{12}{*}{$\begin{array}{l}\text { Networking services } \\
\text { layer }\left(\mathrm{QS}_{5}\right)\end{array}$} & Communication Types[24] & Human to human $(\mathrm{H} 2 \mathrm{H})\left(\mathrm{QS}_{511}\right)$, \\
\hline & $\left(\mathrm{QS}_{51}\right)$ & Human to machine $(\mathrm{H} 2 \mathrm{M})\left(\mathrm{QS}_{512}\right)$ \\
\hline & & Machine to machine $(\mathrm{M} 2 \mathrm{M})\left(\mathrm{QS}_{513}\right)$ \\
\hline & & Standardization and efficiency $\left(\mathrm{QS}_{514}\right)$ \\
\hline & Connection Technologies & Message scheduling $\left(\mathrm{QS}_{521}\right)$ \\
\hline & $\left(\mathrm{QS}_{52}\right)$ & Network Protocol $\left(\mathrm{QS}_{522}\right)$ \\
\hline & & Real-time cellular connectivity $\left(\mathrm{QS}_{523}\right)$ \\
\hline & Routing $\left(\mathrm{QS}_{53}\right)$ & Large scale $\left(\mathrm{QS}_{531}\right)$ \\
\hline & & Traffic management and improve the throughput $\left(\mathrm{QS}_{532}\right)$ \\
\hline & & Range and coverage $\left(\mathrm{QS}_{533}\right)$ \\
\hline & & Routing paths across the IoT nodes $\left(\mathrm{QS}_{534}\right)$ \\
\hline & & Mobility and location $\left(\mathrm{QS}_{535}\right)$ \\
\hline
\end{tabular}

\subsection{Measure $\mathbf{R}$ and $\mathrm{W}$ using linguistic terms}

The performance ratings and the importance weights for service quality attributes are measured using linguistic terms. The concept of linguistic variables to evaluate performance ratings and importance weights are defined in Table 3. These values are to be used directly to evaluate the rating, which 
characterizes the performance of different service quality capabilities, thorough evaluations of overall performance, and weight significance characteristics of QoS, as shown in Table 4.

Table 3. Linguistic variables with fuzzy numbers [18]

\begin{tabular}{|c|c|c|c|}
\hline \multicolumn{2}{|c|}{$\mathrm{R}$} & \multicolumn{2}{|c|}{ W } \\
\hline Linguistic variable & Fuzzy numbers & Linguistic variable & Fuzzy numbers \\
\hline Worst $(\mathrm{Wr})$ & $\left(\begin{array}{llll}0 & 0.5 & 1.5\end{array}\right)$ & Very low (VL) & $(00.050 .15)$ \\
\hline Very poor (VP) & $\left(\begin{array}{lll}1 & 2 & 3\end{array}\right)$ & Low $(\mathrm{L})$ & $\left(\begin{array}{lll}0.1 & 0.2 & 0.3\end{array}\right)$ \\
\hline Poor $(\mathrm{P})$ & $(23.55)$ & Fairly low (FL) & $\left(\begin{array}{lll}0.2 & 0.35 & 0.5\end{array}\right)$ \\
\hline Fair (F) & (3 57 ) & Medium (M) & $\left(\begin{array}{lll}0.3 & 0.5 & 0.7\end{array}\right)$ \\
\hline Good (G) & (5 6.58$)$ & Fairly high $(\mathrm{FH})$ & (0.5 0.650 .8$)$ \\
\hline Very good (VG) & $(789)$ & $\operatorname{High}(\mathrm{H})$ & $\left(\begin{array}{lll}0.7 & 0.8 & 0.9\end{array}\right)$ \\
\hline Excellent (E) & $(8.59 .510)$ & Very high (VH) & $(0.850 .951 .0)$ \\
\hline
\end{tabular}

Table 4. Performance of QoS attributes

\begin{tabular}{|c|c|c|c|c|c|c|c|c|c|c|c|c|c|}
\hline$\left(\mathbf{Q S}_{\mathrm{i}}\right)$ & $\left(\mathbf{Q S}_{\mathrm{ij}}\right)$ & $\left(\mathrm{QS}_{\mathrm{ijk}}\right)$ & $\mathbf{w}_{\mathbf{i}}$ & $\mathbf{w}_{\mathrm{ij}}$ & $w_{1 \mathrm{jk}}$ & $\mathbf{R}_{\mathbf{1 j k}}$ & $\left(\mathbf{Q S}_{\mathrm{i}}\right)$ & $\left(\mathbf{Q S}_{\mathrm{ij}}\right)$ & $\left(\mathbf{Q S}_{\mathrm{ijk}}\right)$ & $w_{i}$ & $\mathbf{w}_{\mathrm{ij}}$ & $w_{1 j \mathrm{k}}$ & $\mathbf{R}_{1 \mathrm{jk}}$ \\
\hline \multirow{14}{*}{$\left(\mathbf{Q S}_{\mathbf{1}}\right)$} & $\left(\mathrm{QS}_{11}\right)$ & $\left(\mathrm{QS}_{111}\right)$ & $\mathrm{H}$ & $\mathrm{H}$ & $\mathrm{VH}$ & $\mathrm{VG}$ & $\left(\mathbf{Q S} \mathbf{S}_{\mathbf{4}}\right)$ & $\left(\mathrm{QS}_{41}\right)$ & $\left(\mathrm{QS}_{411}\right)$ & $\mathrm{H}$ & $\mathrm{VH}$ & $\mathrm{VH}$ & VG \\
\hline & & $\left(\mathrm{QS}_{112}\right)$ & & & $\mathrm{H}$ & G & & & $\left(\mathrm{QS}_{412}\right)$ & & & $\mathrm{VH}$ & VG \\
\hline & & $\left(\mathrm{QS}_{113}\right)$ & & & $\mathrm{FH}$ & G & & & $\left(\mathrm{QS}_{413}\right)$ & & & $\mathrm{H}$ & $\mathrm{G}$ \\
\hline & $\left(\mathrm{QS}_{12}\right)$ & & & $\mathrm{H}$ & & & & $\left(\mathrm{QS}_{42}\right)$ & $\left(\mathrm{QS}_{421}\right)$ & & $\mathrm{VH}$ & M & G \\
\hline & & & & & & & & & $\left(\mathrm{QS}_{422}\right)$ & & & $\mathrm{FH}$ & $\mathrm{G}$ \\
\hline & & $\left(\mathrm{QS}_{121}\right)$ & & & M & $\mathrm{F}$ & & & $\left(\mathrm{QS}_{423}\right)$ & & & $\mathrm{FH}$ & G \\
\hline & & $\left(\mathrm{QS}_{122}\right)$ & & & M & G & & & $\left(\mathrm{QS}_{424}\right)$ & & & M & $\mathrm{G}$ \\
\hline & & $\left(\mathrm{QS}_{123}\right)$ & & & M & G & & & $\left(\mathrm{QS}_{425}\right)$ & & & $\mathrm{H}$ & G \\
\hline & & & & & & & & & $\left(\mathrm{QS}_{426}\right)$ & & & $\mathrm{VH}$ & $\mathrm{G}$ \\
\hline & & & & & & & & & $\left(\mathrm{QS}_{427}\right)$ & & & $\mathrm{VH}$ & $\mathrm{G}$ \\
\hline & $\left(\mathrm{QS}_{13}\right)$ & $\left(\mathrm{QS}_{131}\right)$ & & $\mathrm{H}$ & $\mathrm{H}$ & G & & $\left(\mathrm{QS}_{43}\right)$ & $\left(\mathrm{QS}_{431}\right)$ & & $\mathrm{VH}$ & $\mathrm{H}$ & G \\
\hline & & $\left(\mathrm{QS}_{132}\right)$ & & & VH & VG & & & $\left(\mathrm{QS}_{432}\right)$ & & & $\mathrm{FH}$ & $\mathrm{F}$ \\
\hline & & $\left(\mathrm{QS}_{133}\right)$ & & & FH & G & & & $\left(\mathrm{QS}_{433}\right)$ & & & $\mathrm{FH}$ & $\mathrm{F}$ \\
\hline & & $\left(\mathrm{QS}_{134}\right)$ & & & $\mathrm{H}$ & VG & & & $\left(\mathrm{QS}_{434}\right)$ & & & $\mathrm{FH}$ & $\mathrm{G}$ \\
\hline \multirow[t]{17}{*}{$\left(\mathbf{Q S} \mathbf{S}_{\mathbf{2}}\right)$} & $\left(\mathrm{QS}_{21}\right)$ & $\left(\mathrm{OS}_{211}\right)$ & $\mathrm{VH}$ & $\mathrm{H}$ & $\mathrm{H}$ & G & $\left(\mathbf{Q S}_{5}\right)$ & $\left(\mathrm{QS}_{51}\right)$ & $\left(\mathrm{QS}_{511}\right)$ & $\mathrm{H}$ & $\mathrm{H}$ & M & G \\
\hline & & $\begin{array}{l}\left(\mathrm{QS}_{211}\right) \\
\left(\mathrm{OS}_{212}\right)\end{array}$ & & & $\begin{array}{l}H \\
H\end{array}$ & G & & & $\left(\mathrm{QS}_{512}\right)$ & & & M & $\mathrm{G}$ \\
\hline & & $\begin{array}{l}\left(\mathrm{QS}_{212}\right) \\
\left(\mathrm{OS}_{213}\right)\end{array}$ & & & $\begin{array}{l}H \\
H\end{array}$ & G & & & $\left(\mathrm{QS}_{513}\right)$ & & & M & G \\
\hline & & $(\mathrm{QS} 213)$ & & & П & U & & & $\left(\mathrm{QS}_{514}\right)$ & & & $\mathrm{FH}$ & G \\
\hline & $\left(\mathrm{QS}_{22}\right)$ & $\left(\mathrm{QS}_{221}\right)$ & & VH & $\mathrm{H}$ & $\mathrm{F}$ & & $\left(\mathrm{QS}_{52}\right)$ & $\left(\mathrm{QS}_{521}\right)$ & & $\mathrm{H}$ & VH & VG \\
\hline & & $\left(\mathrm{QS}_{222}\right)$ & & & FH & G & & & $\left(\mathrm{QS}_{522}\right)$ & & & $\mathrm{H}$ & VG \\
\hline & & $\left(\mathrm{QS}_{223}\right)$ & & & $\mathrm{VH}$ & VG & & & $\left(\mathrm{QS}_{523}\right.$ & & & $\mathrm{H}$ & VG \\
\hline & & $\left(\mathrm{QS}_{224}\right)$ & & & VH & VG & & & & & & & \\
\hline & & $\left(\mathrm{QS}_{225}\right)$ & & & $\mathrm{VH}$ & VG & & & & & & & \\
\hline & $\left(\mathrm{QS}_{23}\right)$ & $\left(\mathrm{QS}_{231}\right)$ & & $\mathrm{H}$ & FH & VG & & $\left(\mathrm{QS}_{53}\right)$ & $\left(\mathrm{QS}_{531}\right)$ & & $\mathrm{VH}$ & M & G \\
\hline & & $\left(\mathrm{QS}_{232}\right)$ & & & M & $\mathrm{G}$ & & & $\left(\mathrm{QS}_{532}\right)$ & & & $\mathrm{H}$ & VG \\
\hline & & $\left(\mathrm{QS}_{233}\right)$ & & & FH & G & & & $\left(\mathrm{QS}_{533}\right)$ & & & M & VG \\
\hline & & $\left(\mathrm{QS}_{234}\right)$ & & & M & G & & & $\left(\mathrm{QS}_{534}\right)$ & & & $\mathrm{H}$ & VG \\
\hline & & $\left(\mathrm{QS}_{235}\right)$ & & & M & G & & & $\left(\mathrm{QS}_{535}\right)$ & & & $\mathrm{H}$ & G \\
\hline & $\left(\mathrm{QS}_{24}\right)$ & $\left(\mathrm{QS}_{241}\right)$ & & $\mathrm{H}$ & M & G & & & & & & & \\
\hline & & $\left(\mathrm{QS}_{242}\right)$ & & & M & G & & & & & & & \\
\hline & & $\left(\mathrm{QS}_{243}\right)$ & & & $\mathrm{FH}$ & VG & & & & & & & \\
\hline \multirow[t]{9}{*}{$\left(\mathbf{Q S} \mathbf{S}_{3}\right)$} & $\left(\mathrm{QS}_{31}\right)$ & $\left(\mathrm{QS}_{311}\right)$ & $\mathrm{H}$ & VH & $\mathrm{VH}$ & VG & & & & & & & \\
\hline & & $\left(\mathrm{QS}_{312}\right)$ & & & VH & VG & & & & & & & \\
\hline & & $\left(\mathrm{QS}_{313}\right)$ & & & $\mathrm{H}$ & G & & & & & & & \\
\hline & $\left(\mathrm{QS}_{32}\right)$ & $\left(\mathrm{QS}_{321}\right)$ & & $\mathrm{H}$ & M & G & & & & & & & \\
\hline & & $\left(\mathrm{QS}_{322}\right)$ & & & M & G & & & & & & & \\
\hline & & $\left(\mathrm{QS}_{323}\right)$ & & & M & G & & & & & & & \\
\hline & $\left(\mathrm{QS}_{33}\right)$ & $\left(\mathrm{QS}_{331}\right)$ & & $\mathrm{H}$ & $\mathrm{H}$ & G & & & & & & & \\
\hline & & $\left(\mathrm{QS}_{332}\right)$ & & & FH & $\mathrm{F}$ & & & & & & & \\
\hline & & $\left(\mathrm{QS}_{333}\right)$ & & & $\mathrm{H}$ & $\mathrm{F}$ & & & & & & & \\
\hline
\end{tabular}

\subsection{Rounding linguistic terms from ambiguous numbers}

The linguistic value can be manipulated with a fuzzy number [29], by applying the relationship between linguistic terms and fuzzy numbers. As shown in Table 4, linguistic terms are transferred in ambiguous numbers. The results are shown in Table 5.

\subsection{Determine the FQSI using fuzzy weights}

FQSI is an aggregation of fuzzy scores with fuzzy weights, which represents the overall QoS. The fuzzy QoS Level two sustainability criteria index can be calculated using the (5).

$$
Q S_{11}=R_{11}=\left(\begin{array}{lll}
5.8293 & 7.0938 & 8.3704
\end{array}\right)
$$


As a sample, the fuzzy index for the criteria $\left(\mathrm{QS}_{11}\right)$ can be calculated in (12). By applying the same equation, other fuzzy indexes of $\mathrm{QS}_{\mathrm{ij}}$ and QSI are obtained as listed in Table 6. Finally, with applying the (6), the FQSI was calculated as follow:

$\mathrm{FQSI}=\left(\begin{array}{lll}5.4587 & 6.8291 & 8.2101\end{array}\right)$

Table 5. Rounding terms with ambiguous numbers

\begin{tabular}{|c|c|c|c|c|c|c|}
\hline$(\mathrm{QSi})$ & (QSij) & (QSijk) & $\mathbf{w}_{i}$ & $\mathbf{w}_{\mathrm{ij}}$ & $w_{1 j k}$ & $\mathbf{R}_{\mathbf{1 j k}}$ \\
\hline \multirow[t]{10}{*}{$\left(\mathbf{Q S} \mathbf{S}_{1}\right)$} & $\left(\mathrm{QS}_{11}\right)$ & $\left(\mathrm{QS}_{111}\right)$ & $\left(\begin{array}{llll}0.7 & 0.8 & 0.9\end{array}\right)$ & $\left(\begin{array}{llll}0.7 & 0.8 & 0.9\end{array}\right)$ & 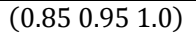 & (789) \\
\hline & & $\left(\mathrm{QS}_{112}\right)$ & & & $(0.70 .80 .9)$ & (5 6.58$)$ \\
\hline & & $\left(\mathrm{QS}_{113}\right)$ & & & $\left(\begin{array}{lll}0.5 & 0.65 & 0.8\end{array}\right)$ & (5 6.58$)$ \\
\hline & $\left(\mathrm{QS}_{12}\right)$ & $\left(\mathrm{QS}_{121}\right)$ & & $\left(\begin{array}{lll}0.7 & 0.8 & 0.9\end{array}\right)$ & $\left(\begin{array}{lll}0.3 & 0.5 & 0.7\end{array}\right)$ & $(357)$ \\
\hline & & $\left(\mathrm{QS}_{122}\right)$ & & & $\left(\begin{array}{llll}0 & 0.3 & 0.5 & 0.7\end{array}\right)$ & (5 6.58$)$ \\
\hline & & $\left(\mathrm{QS}_{123}\right)$ & & & $\left(\begin{array}{lll}0.3 & 0.5 & 0.7\end{array}\right)$ & (5 6.5 8) \\
\hline & $\left(\mathrm{QS}_{13}\right)$ & $\left(\mathrm{QS}_{131}\right)$ & & $\left(\begin{array}{llll}0.7 & 0.8 & 0.9\end{array}\right)$ & $\left(\begin{array}{llll}0.7 & 0.8 & 0.9\end{array}\right)$ & (5 6.58$)$ \\
\hline & & $\left(\mathrm{QS}_{132}\right)$ & & & $(0.850 .951 .0)$ & $(789)$ \\
\hline & & $\left(\mathrm{QS}_{133}\right)$ & & & 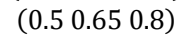 & (5 6.58$)$ \\
\hline & & $\left(\mathrm{QS}_{134}\right)$ & & & $\left(\begin{array}{lll}0.7 & 0.8 & 0.9\end{array}\right)$ & (7 89 ) \\
\hline \multirow[t]{16}{*}{$\left(\mathbf{Q S}_{2}\right)$} & $\left(\mathrm{QS}_{21}\right)$ & $\left(\mathrm{QS}_{211}\right)$ & 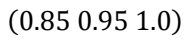 & $\left(\begin{array}{lll}0.7 & 0.8 & 0.9\end{array}\right)$ & $\left(\begin{array}{lll}0.7 & 0.8 & 0.9\end{array}\right)$ & (5 6.58$)$ \\
\hline & & $\left(\mathrm{QS}_{212}\right)$ & & & $\left(\begin{array}{llll}0.7 & 0.8 & 0.9\end{array}\right)$ & (5 6.58 ) \\
\hline & & $\left(\mathrm{QS}_{213}\right)$ & & & $\left(\begin{array}{llll}0.7 & 0.8 & 0.9\end{array}\right)$ & (5 6.58 ) \\
\hline & $\left(\mathrm{QS}_{22}\right)$ & $\left(\mathrm{QS}_{221}\right)$ & & $\left(\begin{array}{lll}0.85 & 0.95 & 1.0\end{array}\right)$ & $\left(\begin{array}{llll}0.7 & 0.8 & 0.9\end{array}\right)$ & (35 7) \\
\hline & & $\left(\mathrm{QS}_{222}\right)$ & & & $\left(\begin{array}{lll}0.5 & 0.65 & 0.8\end{array}\right)$ & (5 6.58$)$ \\
\hline & & $\left(\mathrm{QS}_{223}\right)$ & & & $(0.850 .951 .0)$ & (7 89 9) \\
\hline & & $\left(\mathrm{QS}_{224}\right)$ & & & $(0.850 .951 .0)$ & (7 89 ) \\
\hline & & $\left(\mathrm{QS}_{225}\right)$ & & & $(0.850 .951 .0)$ & (7 89 9) \\
\hline & $\left(\mathrm{QS}_{23}\right)$ & $\left(\mathrm{QS}_{231}\right)$ & & $\left(\begin{array}{lll}0.7 & 0.8 & 0.9\end{array}\right)$ & $\left(\begin{array}{lll}0.5 & 0.65 & 0.8)\end{array}\right)$ & (7 89 ) \\
\hline & & $\left(\mathrm{QS}_{232}\right)$ & & & $\left(\begin{array}{llll}0.3 & 0.5 & 0.7\end{array}\right)$ & (5 6.58 ) \\
\hline & & $\left(\mathrm{QS}_{233}\right)$ & & & $(0.50 .650 .8)$ & (5 6.58$)$ \\
\hline & & $\left(\mathrm{QS}_{234}\right)$ & & & $\left(\begin{array}{llll}0.3 & 0.5 & 0.7\end{array}\right)$ & (5 6.58 ) \\
\hline & & $\left(\mathrm{QS}_{235}\right)$ & & & $\left(\begin{array}{lll}0.3 & 0.5 & 0.7\end{array}\right)$ & (5 6.58$)$ \\
\hline & $\left(\mathrm{QS}_{24}\right)$ & $\left(\mathrm{QS}_{241}\right)$ & & $\left(\begin{array}{llll}0.7 & 0.8 & 0.9\end{array}\right)$ & $\left(\begin{array}{llll}0.3 & 0.5 & 0.7\end{array}\right)$ & (5 6.58$)$ \\
\hline & & $\left(\mathrm{QS}_{242}\right)$ & & & $\left(\begin{array}{lll}0.3 & 0.5 & 0.7\end{array}\right)$ & (5 6.58$)$ \\
\hline & & $\left(\mathrm{QS}_{243}\right)$ & & & 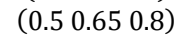 & $(789)$ \\
\hline \multirow[t]{9}{*}{$\left(\mathbf{Q S} \mathbf{S}_{3}\right)$} & $\left(\mathrm{QS}_{31}\right)$ & $\left(\mathrm{QS}_{311}\right)$ & $\left(\begin{array}{lll}0.7 & 0.8 & 0.9\end{array}\right)$ & $(0.850 .951 .0)$ & $(0.850 .951 .0)$ & (7 89 ) \\
\hline & & $\left(\mathrm{QS}_{312}\right)$ & & & (0.85 0.951 .0$)$ & (7 89 ) \\
\hline & & $\left(\mathrm{QS}_{313}\right)$ & & & $\left(\begin{array}{llll}0.7 & 0.8 & 0.9\end{array}\right)$ & (5 6.58 8) \\
\hline & $\left(\mathrm{QS}_{32}\right)$ & $\left(\mathrm{QS}_{321}\right)$ & & $\left(\begin{array}{lll}0.7 & 0.8 & 0.9\end{array}\right)$ & $\left(\begin{array}{llll}0 & 0.3 & 0.5 & 0.7\end{array}\right)$ & (5 6.58$)$ \\
\hline & & $\left(\mathrm{QS}_{322}\right)$ & & & $\left(\begin{array}{lll}0.3 & 0.5 & 0.7\end{array}\right)$ & (5 6.5 8) \\
\hline & & $\left(\mathrm{QS}_{323}\right)$ & & & $\left(\begin{array}{lll}0.3 & 0.5 & 0.7\end{array}\right)$ & (5 6.5 8) \\
\hline & $\left(\mathrm{QS}_{33}\right)$ & $\left(\mathrm{QS}_{331}\right)$ & & (0.7 0.8 0.9 & $\left(\begin{array}{lll}0.7 & 0.8 & 0.9\end{array}\right)$ & (5 6.58$)$ \\
\hline & & $\left(\mathrm{QS}_{332}\right)$ & & & $\left(\begin{array}{lll}0.5 & 0.65 & 0.8)\end{array}\right)$ & $(357)$ \\
\hline & & $\left(\mathrm{QS}_{333}\right)$ & & & $\left(\begin{array}{llll}0.7 & 0.8 & 0.9\end{array}\right)$ & $(357)$ \\
\hline \multirow[t]{14}{*}{$\left(\mathbf{Q S}_{4}\right)$} & $\left(\mathrm{QS}_{41}\right)$ & $\left(\mathrm{QS}_{411}\right)$ & $\left(\begin{array}{lll}0.7 & 0.8 & 0.9\end{array}\right)$ & $(0.850 .951 .0)$ & $(0.850 .951 .0)$ & $(7899)$ \\
\hline & & $\left(\mathrm{QS}_{412}\right)$ & & & $(0.850 .951 .0)$ & (789) \\
\hline & & $\left(\mathrm{QS}_{413}\right)$ & & & $\left(\begin{array}{lll}0.7 & 0.8 & 0.9\end{array}\right)$ & (5 6.58 ) \\
\hline & $\left(\mathrm{QS}_{42}\right)$ & $\left(\mathrm{QS}_{421}\right)$ & & (0.85 0.951 .0$)$ & $\left(\begin{array}{lll}0.3 & 0.5 & 0.7\end{array}\right)$ & (5 6.5 8) \\
\hline & & $\left(\mathrm{QS}_{422}\right)$ & & & 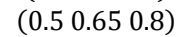 & (5 6.58$)$ \\
\hline & & $\left(\mathrm{QS}_{423}\right)$ & & & (0.5 0.650 .8$)$ & (5 6.58 ) \\
\hline & & $\left(\mathrm{QS}_{424}\right)$ & & & $\left(\begin{array}{lll}0.3 & 0.5 & 0.7\end{array}\right)$ & (5 6.58$)$ \\
\hline & & $\left(\mathrm{QS}_{425}\right)$ & & & $\left(\begin{array}{llll}0.7 & 0.8 & 0.9\end{array}\right)$ & (5 6.58 ) \\
\hline & & $\left(\mathrm{QS}_{426}\right)$ & & & $(0.850 .951 .0)$ & (5 6.58$)$ \\
\hline & & $\left(\mathrm{QS}_{427}\right)$ & & & $(0.850 .951 .0)$ & (5 6.58 ) \\
\hline & $\left(\mathrm{QS}_{43}\right)$ & $\left(\mathrm{QS}_{431}\right)$ & & $(0.850 .951 .0)$ & $\left(\begin{array}{lll}0.7 & 0.8 & 0.9\end{array}\right)$ & (5 6.58$)$ \\
\hline & & $\left(\mathrm{QS}_{432}\right)$ & & & (0.5 0.650 .8$)$ & (35 7) \\
\hline & & $\left(\mathrm{QS}_{433}\right)$ & & & 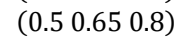 & (35 7) \\
\hline & & $\left(\mathrm{QS}_{434}\right)$ & & & $\left(\begin{array}{lll}0.5 & 0.65 & 0.8)\end{array}\right)$ & (5 6.58 ) \\
\hline \multirow[t]{12}{*}{$\left(\mathbf{Q S} \mathbf{S}_{5}\right)$} & $\left(\mathrm{QS}_{51}\right)$ & $\left(\mathrm{QS}_{511}\right)$ & $\left(\begin{array}{lll}0.7 & 0.8 & 0.9\end{array}\right)$ & $\left(\begin{array}{lll}0.7 & 0.8 & 0.9\end{array}\right)$ & $\left(\begin{array}{lll}0.3 & 0.5 & 0.7\end{array}\right)$ & (5 6.58$)$ \\
\hline & & $\left(\mathrm{QS}_{512}\right)$ & & & $\left(\begin{array}{llll}0.3 & 0.5 & 0.7\end{array}\right)$ & (5 6.58 ) \\
\hline & & $\left(\mathrm{QS}_{513}\right)$ & & & $\left(\begin{array}{lll}0.3 & 0.5 & 0.7\end{array}\right)$ & (5 6.58$)$ \\
\hline & & $\left(\mathrm{QS}_{514}\right)$ & & & $\left(\begin{array}{lll}0.5 & 0.65 & 0.8)\end{array}\right.$ & (5 6.5 8) \\
\hline & $\left(\mathrm{QS}_{52}\right)$ & $\left(Q_{521}\right)$ & & $\left(\begin{array}{lll}0.7 & 0.8 & 0.9\end{array}\right)$ & $(0.850 .951 .0)$ & (7 89 ) \\
\hline & & $\left(\mathrm{QS}_{522}\right)$ & & & $\left(\begin{array}{llll}0.7 & 0.8 & 0.9\end{array}\right)$ & (789) \\
\hline & & $\left(\mathrm{QS}_{523}\right)$ & & & $\left(\begin{array}{lll}0.7 & 0.8 & 0.9\end{array}\right)$ & (7 89 ) \\
\hline & $\left(\mathrm{QS}_{53}\right)$ & $\left(\mathrm{QS}_{531}\right)$ & & 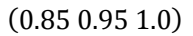 & $\left(\begin{array}{llll}0.3 & 0.5 & 0.7\end{array}\right)$ & (5 6.58$)$ \\
\hline & & $\left(\mathrm{QS}_{532}\right)$ & & & $\left(\begin{array}{lll}0.7 & 0.8 & 0.9\end{array}\right)$ & (789) \\
\hline & & $\left(\mathrm{QS}_{533}\right)$ & & & $\left(\begin{array}{lll}0.3 & 0.5 & 0.7\end{array}\right)$ & (7 89 ) \\
\hline & & $\left(\mathrm{QS}_{534}\right)$ & & & $\left(\begin{array}{lll}0.7 & 0.8 & 0.9\end{array}\right)$ & (7 89 ) \\
\hline & & $\left(\mathrm{QS}_{535}\right)$ & & & $\left(\begin{array}{llll}0.7 & 0.8 & 0.9\end{array}\right)$ & (5 6.58$)$ \\
\hline
\end{tabular}




\begin{tabular}{ccccccccc}
\multicolumn{8}{c}{ Table 6. Fuzzy indexes } \\
\hline $\mathrm{QS}_{i}$ & & $\mathrm{R}_{\mathrm{i}}$ & & $\mathrm{QS}_{\mathrm{ij}}$ & & $\mathrm{R}_{\mathrm{ij}}$ \\
\hline $\mathrm{QS}_{1}$ & $(5.4300$ & 6.8047 & $8.1883)$ & $\mathrm{QS}_{11}$ & $(5.8293$ & 7.0938 & $8.3704)$ \\
& & & & $\mathrm{QS}_{12}$ & $(4.3333$ & 6.0000 & $7.6667)$ \\
& & & & $\mathrm{QS}_{13}$ & $(6.1273$ & 7.3203 & $8.5278)$ \\
$\mathrm{QS}_{2}$ & $(5.6055$ & 6.9136 & $8.2567)$ & $\mathrm{QS}_{21}$ & $(5.0000$ & 6.5000 & $8.0000)$ \\
& & & & $\mathrm{QS}_{22}$ & $(5.9867$ & 7.2151 & $8.4468)$ \\
& & & & $\mathrm{QS}_{23}$ & $(5.5263$ & 6.8482 & $8.2162)$ \\
$\mathrm{QS}_{3}$ & $(4.9711$ & 6.4873 & $7.9954)$ & $\mathrm{QS}_{24}$ & $(5.9091$ & 7.0909 & $8.3636)$ \\
& & & & $\mathrm{QS}_{31}$ & $(6.1765$ & 7.4286 & $8.6400)$ \\
& & & & $\mathrm{QS}_{32}$ & $(5.0000$ & 6.5000 & $8.0000)$ \\
$\mathrm{QS}_{4}$ & $(5.1692$ & 6.6155 & $8.0683)$ & $\mathrm{QS}_{33}$ & $(3.7368$ & 5.5333 & $7.3462)$ \\
& & & & $\mathrm{QS}_{41}$ & $(6.4167$ & 7.5556 & $8.6897)$ \\
& & & & $\mathrm{QS}_{42}$ & $(5.0000$ & 6.5000 & $8.0000)$ \\
$\mathrm{QS}_{5}$ & $(6.0864$ & 7.3088 & $8.5366)$ & $\mathrm{QS}_{43}$ & $(4.0909$ & 5.7909 & $7.5152)$ \\
& & & & $\mathrm{QS}_{51}$ & $(5.0000$ & 6.5000 & $8.0000)$ \\
& & & & $\mathrm{QS}_{52}$ & $(7.0000$ & 8.0000 & $9.0000)$ \\
& & & & $\mathrm{QS}_{53}$ & $(6.2593$ & 7.4265 & $8.6098)$ \\
\hline
\end{tabular}

\subsection{Determine the euclidean distance to match FQSI with the approximate QoS level}

To identify the level of sustainability. The obtained FQSI can be matched with linguistic terms by using the (7). The natural linguistic set L [7], where $\mathrm{L}=$ [Extremely SQoS [EQS (7, 8.5, 10)]; Very SQoS [VQS (5.5, 7, 8.5)]; Medium SQoS [MQS (3.5, 5, 6.5)]; Fairly SQoS [FQS (1.5, 3, 4.5)]; Slowly SQoS [SQS $(0,1.5,3)]\}$. Euclidean distance D is obtained from the FQSI for each member of the L-set and is calculated as:

$$
\begin{aligned}
& \mathrm{D}(\mathrm{FQSI}, \mathrm{EQS})=2.893 \\
& \mathrm{D}(\mathrm{FQSI}, \mathrm{VQS})=0.3391 \\
& \mathrm{D}(\mathrm{FQSI}, \mathrm{MQS})=3.1791 \\
& \mathrm{D}(\mathrm{FQSI}, \mathrm{FQS})=6.6407 \\
& \mathrm{D}(\mathrm{FQSI}, \mathrm{SQS})=9.2381
\end{aligned}
$$

\subsection{Matching FQSI}

To the appropriate level After the distances are obtained, the minimum distance (D) is chosen as the current status as:

$$
\min \{\mathrm{D}(\mathrm{FQSI}, \mathrm{EQS}), \mathrm{D}(\mathrm{FQSI}, \mathrm{VQS}), \mathrm{D}(\mathrm{FQSI}, \mathrm{MQS}), \mathrm{D}(\mathrm{FQSI}, \mathrm{FQS}), \mathrm{D}(\mathrm{FQSI}, \mathrm{SQS})\}=\mathrm{D}(\mathrm{FQSI}, \mathrm{VQS})
$$

thus, by matching the minimum $\mathrm{D}$ with a linguistic label, the sustainable quality of service index level can be identified as «very sustainable QoS», as shown in Figure 2.

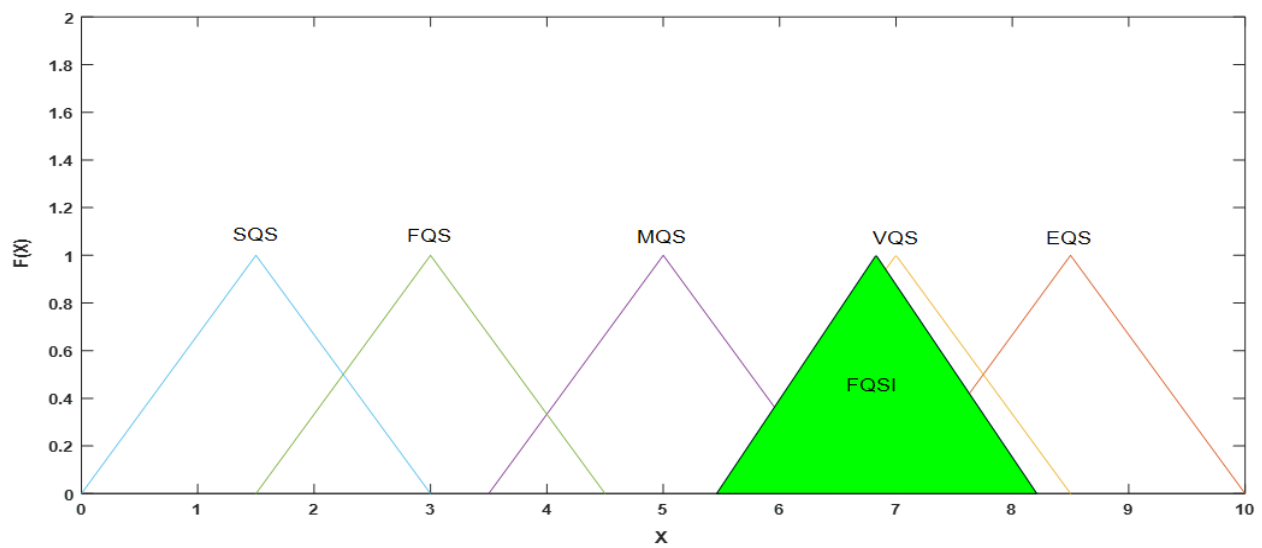

Figure 2. Linguistic levels to match FQSI

\subsection{Analysis the main obstacles}

Regarding the efficiency of the QoS measurement method, the obtained fuzzy SQoS index has been validated using conventional crisp. The result obtained by applying the fuzzy and traditional approaches gives the same results as shown in Table 7 , by using the conventional crispy technique, the service quality index was found at 6.84 and the resulting QSI was validated as shown in Table 8. 
Table 7. A computation index of QoS attributes using crisp approach

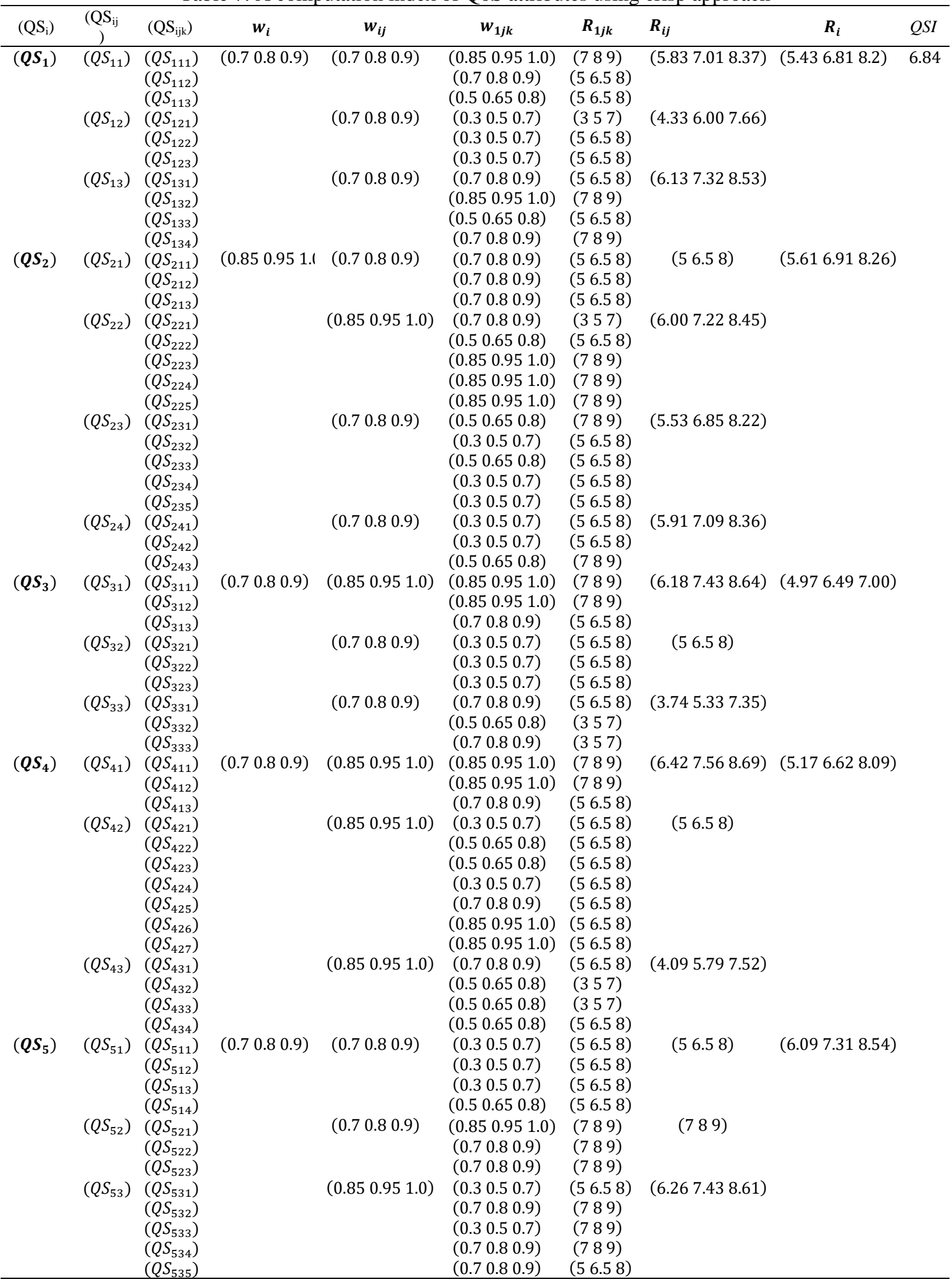

Regarding the efficiency of the SQoS index measurement method. Table 8 monitors the result obtained by applying the fuzzy and conventional approaches, leading to similar conclusions. The FQSI is generated by the fuzzy logic approach and expressed in terms of value ranges. This rating can give an overall solution of the relevant possibility and ensure that the decision taken in the selection process is correct. In addition, it gives decision-makers great flexibility in making decisions making. 
Table 8. A comparison between two approaches, fuzzy and crisp logic

\begin{tabular}{|c|c|c|c|c|c|}
\hline Approach & QoS sust & ainabi & ty index & Range & Linguistic labeling \\
\hline $\begin{array}{c}\text { Fuzy logic } \\
\text { Crisp Approach }\end{array}$ & $(5.46$ & $\begin{array}{l}6.83 \\
6.84\end{array}$ & 8.21) & 2.75 & very sustainable QoS \\
\hline
\end{tabular}

According to the evaluation, there were obstacles that could influence the SQoS. To identify these obstacles for improving the sustainability level of QoS [16], we used FPII, as indicated in the (8) and (9), which combines $\mathrm{R}$ and $\mathrm{W}$ of each sustainability service. For example,

$$
\mathrm{FPII}_{111}=\left(\begin{array}{l}
1.050 .40 .0
\end{array}\right)
$$

then, the remaining attributes of the FPII are calculated by the same method cited above [17], as shown in Table 9. The ranking of the fuzzy number is based on a centroid method for the membership function $(a, b, c)$ (10), where a, b, c, they are the lower, middle, and higher numbers than the triangular fuzzy number.

\section{Ranking Score $=0.44$}

To identify the few critical barriers, experts set a value of 0.93 as a threshold to distinguish critical obstacles to be improved as shown in Figure 3. Subsequently, as shown in Table 9, depending on the threshold value we found that there are 14 values less than or equal to the threshold value. These values represent the weaker features of the services in the system, and therefore these fourteen attributes represent significant contributions and should be improved to achieve SQoS. In addition, in Figure 4, an illustrative comparison between the number of total services and the results obtained for each of the layers of quality of service. In terms of the rate of high and low services. The purpose of this comparison is to clarify and help developers to decide which area or layer to focus on in order to improve and develop.

As shown in Figure 5, based on the assessment method approach in the smart home system, the obtained result has two principal limited values, which are the values of the low services that are closest to optimization, and the values of the worst lower services. This assessment provides an illustration for determining the QoS in order to improve and ensure the sustainability of services. In addition, FPII of QoS was computed to improve the weaker services areas that have been identified. $78 \%$ of the best stable services were found, which is an encouraging percentage compared to the ratio of the weakest services estimated at $22 \%$ as shown in Figure 6. Through these percentages, we conclude that the proposed approach is highly capable of evaluating QoS. In addition, it ensures the continuity of the system by identifying the least effective services and the sustainability of high services.

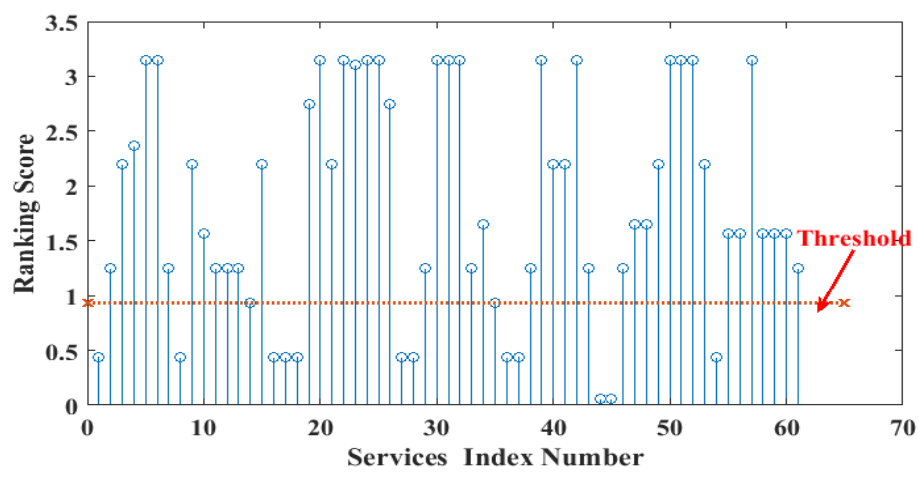

Figure 3. Ranking scores for services and threshold value of low services

As shown in the Table 10, the weakest services were classified. Some modifications and amelioration were made to improve these services and obstacles. For example, data control management applications, and regular maintenance could be increase data confidentiality and improve operational efficiency to develop a remote control system. Installations of many IoT items could be improved, by a good identification of our need for IoT elements. The development of a trusted operator for mixed-use users could improve the independent systems of users. 
Table 9. The fuzzy performance index of QoS attributes

\begin{tabular}{|c|c|c|c|c|c|}
\hline (QSijk) & $w_{1 \mathrm{jk}}$ & $\mathbf{w}_{\mathbf{1 j k}}^{\prime}$ & $R_{1 j k}$ & FPII & Ranking score \\
\hline$\left(\mathbf{Q S}_{111}\right)$ & $\left(\begin{array}{llll}0.85 & 0.95 & 1.0\end{array}\right)$ & $\left(\begin{array}{llll}0.15 & 0.05 & 0.0\end{array}\right)$ & $(789)$ & $\left(\begin{array}{lll}1.05 & 0.4 & 0.0\end{array}\right)$ & 0.44 \\
\hline$\left(\mathbf{Q S}_{112}\right)$ & $\left(\begin{array}{lll}0.7 & 0.8 & 0.9\end{array}\right)$ & $\left(\begin{array}{lll}0.3 & 0.2 & 0.1\end{array}\right)$ & (5 6.5 8) & (1.5 1.30 .8$)$ & 1.25 \\
\hline$\left(\mathbf{Q S}_{113}\right)$ & (0.5 0.650 .8$)$ & $\left(\begin{array}{lll}0.5 & 0.35 & 0.2\end{array}\right)$ & (5 6.5 8) & (2.5 2.28 1.6) & 2.20 \\
\hline$\left(\mathbf{Q S}_{121}\right)$ & $\left(\begin{array}{lll}0.3 & 0.5 & 0.7\end{array}\right)$ & $\left(\begin{array}{lll}0.7 & 0.5 & 0.3)\end{array}\right.$ & $(357)$ & $\left(\begin{array}{lll}2.1 & 2.5 & 2.1)\end{array}\right.$ & 2.37 \\
\hline$\left(\mathbf{Q S}_{122}\right)$ & $\left(\begin{array}{lll}0.3 & 0.5 & 0.7\end{array}\right)$ & $\left(\begin{array}{lll}0.7 & 0.5 & 0.3\end{array}\right)$ & (5 6.5 8) & (3.5 3.25 2.4) & 3.15 \\
\hline$\left(\mathbf{Q S}_{123}\right)$ & $\left(\begin{array}{lll}0.3 & 0.5 & 0.7\end{array}\right)$ & $\left(\begin{array}{lll}0.7 & 0.5 & 0.3\end{array}\right)$ & (5 6.5 8) & (3.5 3.25 2.4) & 3.15 \\
\hline$\left(\mathbf{Q S}_{131}\right)$ & $\left(\begin{array}{lll}0.7 & 0.8 & 0.9\end{array}\right)$ & $\left(\begin{array}{lll}0.3 & 0.2 & 0.1\end{array}\right)$ & (5 6.5 8) & (1.5 1.30 .8$)$ & 1.25 \\
\hline$\left(\mathbf{Q S}_{132}\right)$ & $\left(\begin{array}{llll}0.85 & 0.95 & 1.0\end{array}\right)$ & $\left(\begin{array}{lll}0.15 & 0.05 & 0.0\end{array}\right)$ & $(789)$ & (1.05 0.40 .0$)$ & 0.44 \\
\hline$\left(\mathbf{Q S}_{133}\right)$ & (0.5 0.650 .8$)$ & $\left(\begin{array}{lll}0.5 & 0.35 & 0.2\end{array}\right)$ & (5 6.5 8) & (2.5 2.28 1.6) & 2.20 \\
\hline$\left(\mathbf{Q S}_{134}\right)$ & $\left(\begin{array}{lll}0.7 & 0.8 & 0.9\end{array}\right)$ & $\left(\begin{array}{lll}0.3 & 0.2 & 0.1\end{array}\right)$ & $(789)$ & $\left(\begin{array}{lll}2.1 & 1.6 & 0.9\end{array}\right)$ & 1.57 \\
\hline$\left(\mathbf{Q S}_{211}\right)$ & $\left(\begin{array}{lll}0.7 & 0.8 & 0.9\end{array}\right)$ & $\left(\begin{array}{lll}0.3 & 0.2 & 0.1\end{array}\right)$ & (5 6.5 8) & (1.5 1.30 .8$)$ & 1.25 \\
\hline$\left(\mathbf{Q S}_{212}\right)$ & $\left(\begin{array}{lll}0.7 & 0.8 & 0.9\end{array}\right)$ & $\left(\begin{array}{lll}0.3 & 0.2 & 0.1\end{array}\right)$ & (5 6.5 8) & (1.5 1.30 .8$)$ & 1.25 \\
\hline$\left(\mathbf{Q S}_{213}\right)$ & $\left(\begin{array}{lll}0.7 & 0.8 & 0.9)\end{array}\right)$ & $\left(\begin{array}{lll}0.3 & 0.2 & 0.1\end{array}\right)$ & (5 6.5 8) & (1.5 1.30 .8$)$ & 1.25 \\
\hline$\left(\mathbf{Q S}_{221}\right)$ & $\left(\begin{array}{lll}0.7 & 0.8 & 0.9\end{array}\right)$ & $\left(\begin{array}{lll}0.3 & 0.2 & 0.1\end{array}\right)$ & $(357)$ & (0.9 1.00 .7$)$ & 0.93 \\
\hline$\left(\mathbf{Q S}_{222}\right)$ & (0.5 0.650 .8$)$ & $\left(\begin{array}{lll}0.5 & 0.35 & 0.2\end{array}\right)$ & (5 6.5 8) & (2.5 2.28 1.6) & 2.20 \\
\hline$\left(\mathbf{Q S}_{223}\right)$ & 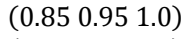 & $\left(\begin{array}{lll}0.15 & 0.05 & 0.0\end{array}\right)$ & $(789)$ & $\left(\begin{array}{lll}1.05 & 0.4 & 0.0\end{array}\right)$ & 0.44 \\
\hline$\left(\mathbf{Q S}_{224}\right)$ & $\left(\begin{array}{lll}0.85 & 0.95 & 1.0\end{array}\right)$ & $\left(\begin{array}{lll}0.15 & 0.05 & 0.0\end{array}\right)$ & (7 89 ) & (1.05 0.40 .0$)$ & 0.44 \\
\hline$\left(\mathbf{Q S}_{225}\right)$ & $\left(\begin{array}{lll}0.85 & 0.95 & 1.0\end{array}\right)$ & $\left(\begin{array}{lll}0.15 & 0.05 & 0.0\end{array}\right)$ & (7 89 ) & (1.05 0.40 .0$)$ & 0.44 \\
\hline$\left(\mathbf{Q S} \mathbf{S}_{231}\right)$ & (0.5 0.650 .8$)$ & $\left(\begin{array}{lll}0.5 & 0.35 & 0.2\end{array}\right)$ & $(789)$ & (3.5 2.8 1.8) & 2.75 \\
\hline$\left(\mathbf{Q S}_{232}\right)$ & $\left(\begin{array}{lll}0.3 & 0.5 & 0.7\end{array}\right)$ & $\left(\begin{array}{lll}0.7 & 0.5 & 0.3\end{array}\right)$ & (5 6.5 8) & (3.5 3.25 2.4) & 3.15 \\
\hline$\left(\mathbf{Q S}_{233}\right)$ & (0.5 0.650 .8$)$ & $\left(\begin{array}{lll}0.5 & 0.35 & 0.2\end{array}\right)$ & (5 6.5 8) & (2.5 2.28 1.6) & 2.20 \\
\hline$\left(\mathbf{Q S}_{234}\right)$ & $\left(\begin{array}{lll}0.3 & 0.5 & 0.7\end{array}\right)$ & $\left(\begin{array}{lll}0.7 & 0.5 & 0.3\end{array}\right)$ & (5 6.5 8) & (3.5 3.25 2.4) & 3.15 \\
\hline$\left(\mathbf{Q S}_{235}\right)$ & $\left(\begin{array}{lll}0.3 & 0.5 & 0.7\end{array}\right)$ & $\left(\begin{array}{lll}0.7 & 0.5 & 0.3\end{array}\right)$ & (5 6.5 8) & (3.5 3.25 2.4) & 3.1 \\
\hline$\left(\mathbf{Q S}_{241}\right)$ & $\left(\begin{array}{lll}0.3 & 0.5 & 0.7\end{array}\right)$ & $\left(\begin{array}{lll}0.7 & 0.5 & 0.3\end{array}\right)$ & (5 6.5 8) & (3.5 3.25 2.4) & 3.15 \\
\hline$\left(\mathbf{Q S}_{242}\right)$ & $\left(\begin{array}{lll}0.3 & 0.5 & 0.7\end{array}\right)$ & $\left(\begin{array}{lll}0.7 & 0.5 & 0.3\end{array}\right)$ & (5 6.5 8) & (3.5 3.25 2.4) & 3.15 \\
\hline$\left(\mathbf{Q S}_{243}\right)$ & (0.5 0.650 .8$)$ & $\left(\begin{array}{lll}0.5 & 0.35 & 0.2\end{array}\right)$ & $(789)$ & (3.5 2.8 1.8) & 2.75 \\
\hline$\left(\mathbf{Q S}_{311}\right)$ & $\left(\begin{array}{llll}0.85 & 0.95 & 1.0\end{array}\right)$ & $\left(\begin{array}{lll}0.15 & 0.05 & 0.0\end{array}\right)$ & (7 89) & (1.05 0.40 .0$)$ & 0.44 \\
\hline$\left(\mathbf{Q S}_{312}\right)$ & $\left(\begin{array}{llll}0.85 & 0.95 & 1.0\end{array}\right)$ & $\left(\begin{array}{lll}0.15 & 0.05 & 0.0\end{array}\right)$ & $(789)$ & (1.05 0.40 .0$)$ & 0.44 \\
\hline$\left(\mathbf{Q S}_{313}\right)$ & $\left(\begin{array}{lll}0.7 & 0.8 & 0.9\end{array}\right)$ & $\left(\begin{array}{lll}0.3 & 0.2 & 0.1\end{array}\right)$ & (5 6.5 8) & (1.5 1.30 .8$)$ & 1.25 \\
\hline$\left(\mathbf{Q S}_{321}\right)$ & $\left(\begin{array}{lll}0.3 & 0.5 & 0.7\end{array}\right)$ & $\left(\begin{array}{lll}0.7 & 0.5 & 0.3\end{array}\right)$ & (5 6.5 8) & (3.5 3.25 2.4) & 3.15 \\
\hline$\left(\mathbf{Q S}_{322}\right)$ & $\left(\begin{array}{lll}0.3 & 0.5 & 0.7\end{array}\right)$ & $\left(\begin{array}{lll}0.7 & 0.5 & 0.3\end{array}\right)$ & (5 6.5 8) & (3.5 3.25 2.4) & 3.15 \\
\hline$\left(\mathbf{Q S}_{323}\right)$ & $\left(\begin{array}{lll}0.3 & 0.5 & 0.7\end{array}\right)$ & $\left(\begin{array}{lll}0.7 & 0.5 & 0.3\end{array}\right)$ & (5 6.5 8) & (3.5 3.25 2.4) & 3.15 \\
\hline$\left(\mathbf{Q S}_{331}\right)$ & $\left(\begin{array}{lll}0.7 & 0.8 & 0.9\end{array}\right)$ & $\left(\begin{array}{lll}0.3 & 0.2 & 0.1\end{array}\right)$ & (5 6.5 8) & $\left(\begin{array}{lll}1.5 & 1.3 & 0.8\end{array}\right)$ & 1.25 \\
\hline$\left(\mathbf{Q S}_{332}\right)$ & (0.5 0.650 .8$)$ & $\left(\begin{array}{lll}0.5 & 0.35 & 0.2\end{array}\right)$ & $(357)$ & (1.5 1.75 1.4) & 1.65 \\
\hline$\left(\mathbf{Q S}_{333}\right)$ & $\left(\begin{array}{lll}0.7 & 0.8 & 0.9\end{array}\right)$ & $\left(\begin{array}{lll}0.3 & 0.2 & 0.1\end{array}\right)$ & $(357)$ & $\left(\begin{array}{lll}0.9 & 1.0 & 0.7\end{array}\right)$ & 0.93 \\
\hline$\left(\mathbf{Q S}_{411}\right)$ & $\left(\begin{array}{llll}0.85 & 0.95 & 1.0\end{array}\right)$ & $\left(\begin{array}{lll}0.15 & 0.05 & 0.0\end{array}\right)$ & $(789)$ & $\left(\begin{array}{lll}1.05 & 0.4 & 0.0\end{array}\right)$ & 0.44 \\
\hline$\left(\mathbf{Q S}_{412}\right)$ & 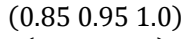 & $\left(\begin{array}{lll}0.15 & 0.05 & 0.0\end{array}\right)$ & (7 89) & $\left(\begin{array}{lll}1.05 & 0.4 & 0.0\end{array}\right)$ & 0.44 \\
\hline$\left(\mathbf{Q S}_{413}\right)$ & $\left(\begin{array}{lll}0.7 & 0.8 & 0.9\end{array}\right)$ & $\left(\begin{array}{lll}0.3 & 0.2 & 0.1\end{array}\right)$ & (5 6.5 8) & (1.5 1.30 .8$)$ & 1.25 \\
\hline$\left(\mathbf{Q S}_{421}\right)$ & $\left(\begin{array}{lll}0.3 & 0.5 & 0.7\end{array}\right)$ & $\left(\begin{array}{lll}0.7 & 0.5 & 0.3\end{array}\right)$ & (5 6.5 8) & (3.5 3.25 2.4) & 3.15 \\
\hline$\left(\mathbf{Q S}_{422}\right)$ & (0.5 0.650 .8$)$ & $\left(\begin{array}{lll}0.5 & 0.35 & 0.2\end{array}\right)$ & (5 6.5 8) & (2.5 2.28 1.6) & 2.20 \\
\hline$\left(\mathbf{Q S}_{423}\right)$ & (0.5 0.650 .8$)$ & $\left(\begin{array}{lll}0.5 & 0.35 & 0.2\end{array}\right)$ & (5 6.5 8) & (2.5 2.28 1.6) & 2.20 \\
\hline$\left(\mathbf{Q S}_{424}\right)$ & ( $\left.\begin{array}{llll}0.3 & 0.5 & 0.7\end{array}\right)$ & $\left(\begin{array}{lll}0.7 & 0.5 & 0.3\end{array}\right)$ & (5 6.5 8) & (3.5 3.25 2.4) & 3.15 \\
\hline$\left(\mathbf{Q S}_{425}\right)$ & ( $\left.\begin{array}{llll}0.7 & 0.8 & 0.9\end{array}\right)$ & $\left(\begin{array}{lll}0.3 & 0.2 & 0.1\end{array}\right)$ & (5 6.5 8) & (1.5 1.30 .8$)$ & 1.25 \\
\hline$\left(\mathbf{Q S}_{426}\right)$ & 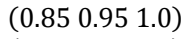 & $\left(\begin{array}{lll}0.15 & 0.05 & 0.0\end{array}\right)$ & (5 6.5 8) & $\left(\begin{array}{lll}0.15 & 0.05 & 0.0\end{array}\right)$ & 0.058 \\
\hline$\left(\mathbf{Q S}_{427}\right)$ & $\left(\begin{array}{llll}0.85 & 0.95 & 1.0\end{array}\right)$ & $\left(\begin{array}{lll}0.15 & 0.05 & 0.0\end{array}\right)$ & (5 6.5 8) & $\left(\begin{array}{lll}0.15 & 0.05 & 0.0\end{array}\right)$ & 0.058 \\
\hline$\left(\mathbf{Q S}_{431}\right)$ & $\left(\begin{array}{lll}0.7 & 0.8 & 0.9\end{array}\right)$ & $\left(\begin{array}{lll}0.3 & 0.2 & 0.1\end{array}\right)$ & (5 6.5 8) & $\left(\begin{array}{lll}1.5 & 1.3 & 0.8\end{array}\right)$ & 1.25 \\
\hline$\left(\mathbf{Q S}_{432}\right)$ & (0.5 0.650 .8$)$ & $\left(\begin{array}{lll}0.5 & 0.35 & 0.2\end{array}\right)$ & (3 57 ) & (1.5 1.75 1.4) & 1.65 \\
\hline$\left(\mathbf{Q S}_{\mathbf{4 3 3}}\right)$ & (0.5 0.650 .8$)$ & $\left(\begin{array}{lll}0.5 & 0.35 & 0.2\end{array}\right)$ & $(357)$ & (1.5 1.75 1.4) & 1.65 \\
\hline$\left(\mathbf{Q S}_{434}\right)$ & (0.5 0.650 .8$)$ & $\left(\begin{array}{lll}0.5 & 0.35 & 0.2\end{array}\right)$ & (5 6.5 8) & $(2.52 .281 .6)$ & 2.20 \\
\hline$\left(\mathbf{Q S}_{511}\right)$ & (0.3 0.5 0.7) & $\left(\begin{array}{lll}0.7 & 0.5 & 0.3\end{array}\right)$ & (5 6.5 8) & (3.5 3.25 2.4) & 3.15 \\
\hline$\left(\mathbf{Q S}_{512}\right)$ & $\left(\begin{array}{lll}0.3 & 0.5 & 0.7\end{array}\right)$ & $\left(\begin{array}{lll}0.7 & 0.5 & 0.3\end{array}\right)$ & (5 6.5 8) & (3.5 3.25 2.4) & 3.15 \\
\hline$\left(\mathbf{Q S}_{513}\right)$ & $\left(\begin{array}{lll}0.3 & 0.5 & 0.7\end{array}\right)$ & $\left(\begin{array}{lll}0.7 & 0.5 & 0.3\end{array}\right)$ & (5 6.5 8) & (3.5 3.25 2.4) & 3.15 \\
\hline$\left(\mathbf{Q S}_{514}\right)$ & (0.5 0.650 .8$)$ & (0.5 0.350 .2$)$ & (5 6.5 8) & (2.5 2.28 1.6) & 2.20 \\
\hline$\left(\mathbf{Q S}_{521}\right)$ & 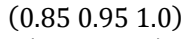 & $\left(\begin{array}{lll}0.15 & 0.05 & 0.0\end{array}\right)$ & $(789)$ & $\left(\begin{array}{lll}1.05 & 0.4 & 0.0\end{array}\right)$ & 0.44 \\
\hline$\left(\mathbf{Q S}_{522}\right)$ & $\left(\begin{array}{lll}0.7 & 0.8 & 0.9\end{array}\right)$ & $\left(\begin{array}{lll}0.3 & 0.2 & 0.1\end{array}\right)$ & $(789)$ & $\left(\begin{array}{lll}2.1 & 1.6 & 0.9\end{array}\right)$ & 1.57 \\
\hline$\left(\mathbf{Q S}_{523}\right)$ & $\left(\begin{array}{lll}0.7 & 0.8 & 0.9\end{array}\right)$ & $\left(\begin{array}{lll}0.3 & 0.2 & 0.1\end{array}\right)$ & (7 89) & $\left(\begin{array}{lll}2.1 & 1.6 & 0.9\end{array}\right)$ & 1.57 \\
\hline$\left(\mathbf{Q S}_{531}\right)$ & $\left(\begin{array}{lll}0.3 & 0.5 & 0.7\end{array}\right)$ & $\left(\begin{array}{lll}0.7 & 0.5 & 0.3\end{array}\right)$ & (5 6.5 8) & (3.5 3.25 2.4) & 3.15 \\
\hline$\left(\mathbf{Q S}_{532}\right)$ & $\left(\begin{array}{lll}0.7 & 0.8 & 0.9\end{array}\right)$ & $\left(\begin{array}{lll}0.3 & 0.2 & 0.1\end{array}\right)$ & $(789)$ & $\left(\begin{array}{lll}2.1 & 1.6 & 0.9\end{array}\right)$ & 1.57 \\
\hline$\left(\mathbf{Q S}_{533}\right)$ & $\left(\begin{array}{lll}0.3 & 0.5 & 0.7\end{array}\right)$ & $\left(\begin{array}{lll}0.7 & 0.5 & 0.3\end{array}\right)$ & $(789)$ & (2.1 1.6 0.9) & 1.57 \\
\hline$\left(\mathbf{Q S}_{534}\right)$ & $\left(\begin{array}{lll}0.7 & 0.8 & 0.9\end{array}\right)$ & $\left(\begin{array}{lll}0.3 & 0.2 & 0.1\end{array}\right)$ & $(789)$ & $\left(\begin{array}{lll}2.1 & 1.6 & 0.9\end{array}\right)$ & 1.57 \\
\hline$\left(\mathbf{Q S}_{535}\right)$ & $\left(\begin{array}{lll}0.7 & 0.8 & 0.9\end{array}\right)$ & $\left(\begin{array}{lll}0.3 & 0.2 & 0.1\end{array}\right)$ & (5 6.5 8) & $\left(\begin{array}{lll}1.5 & 1.3 & 0.8\end{array}\right)$ & 1.25 \\
\hline
\end{tabular}




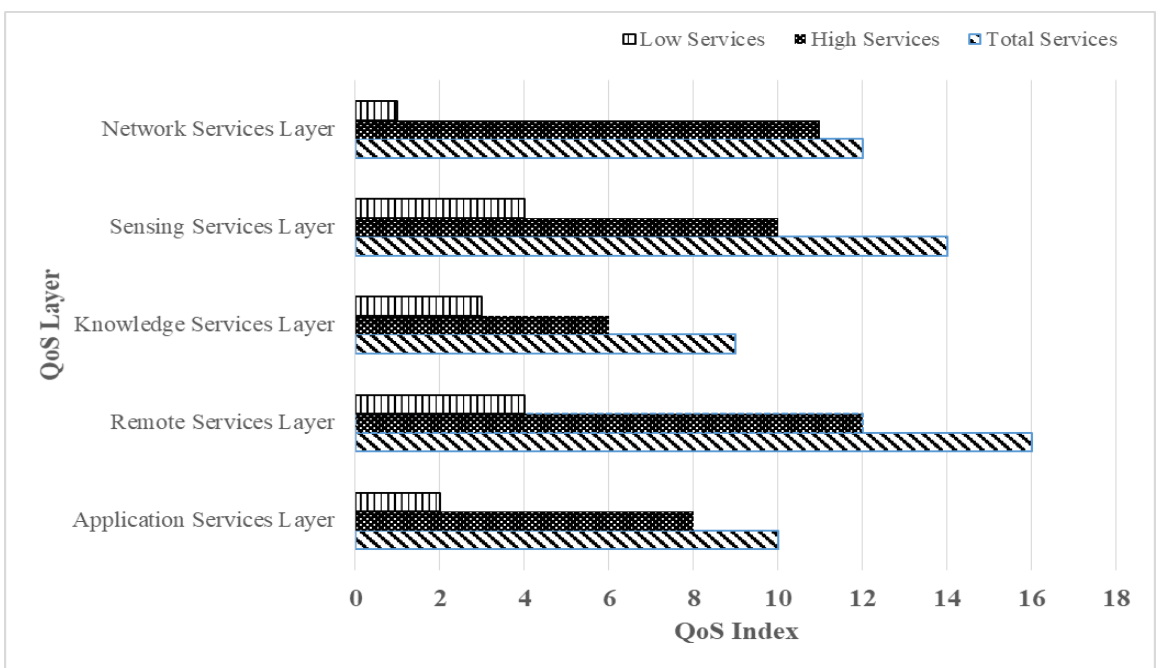

Figure 4. Obstacles and stable services for each layer

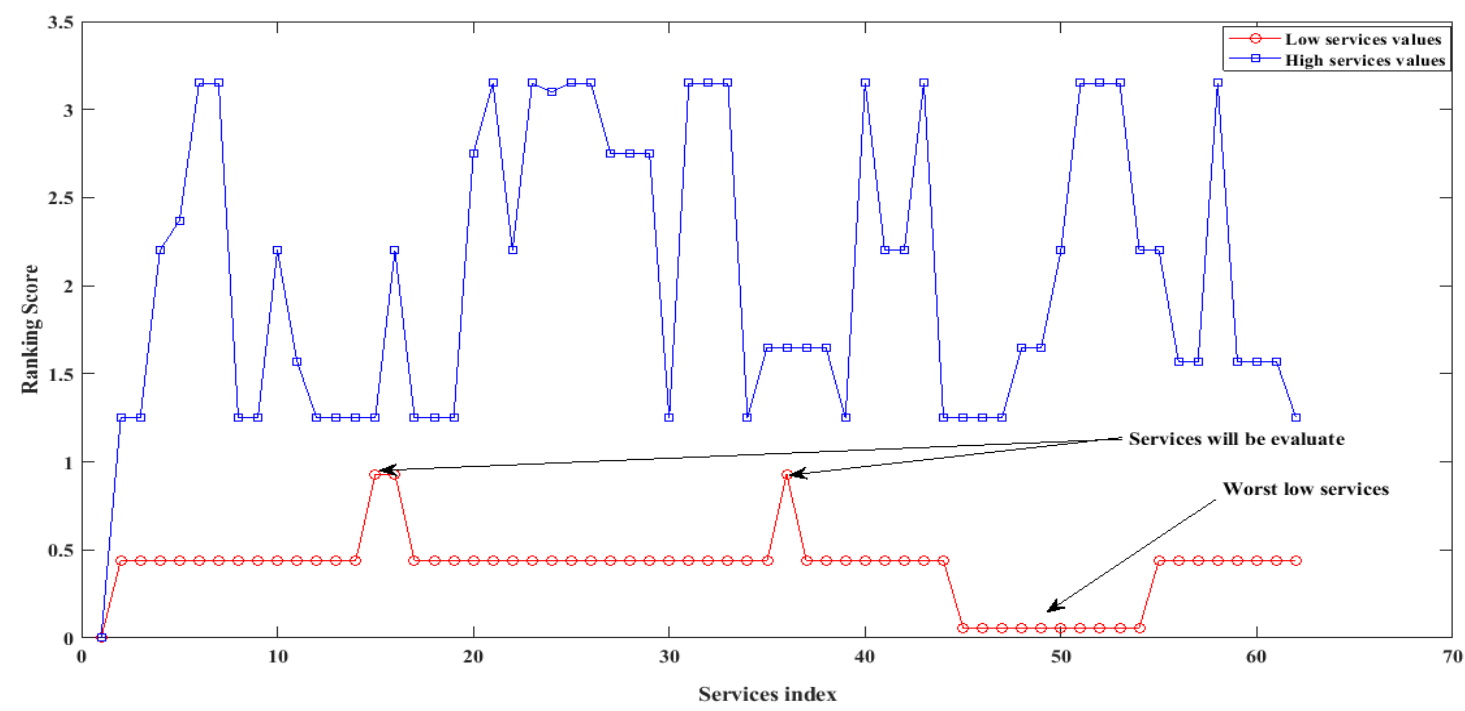

Figure 5. High and low QoS index for a smart home system in 61 services

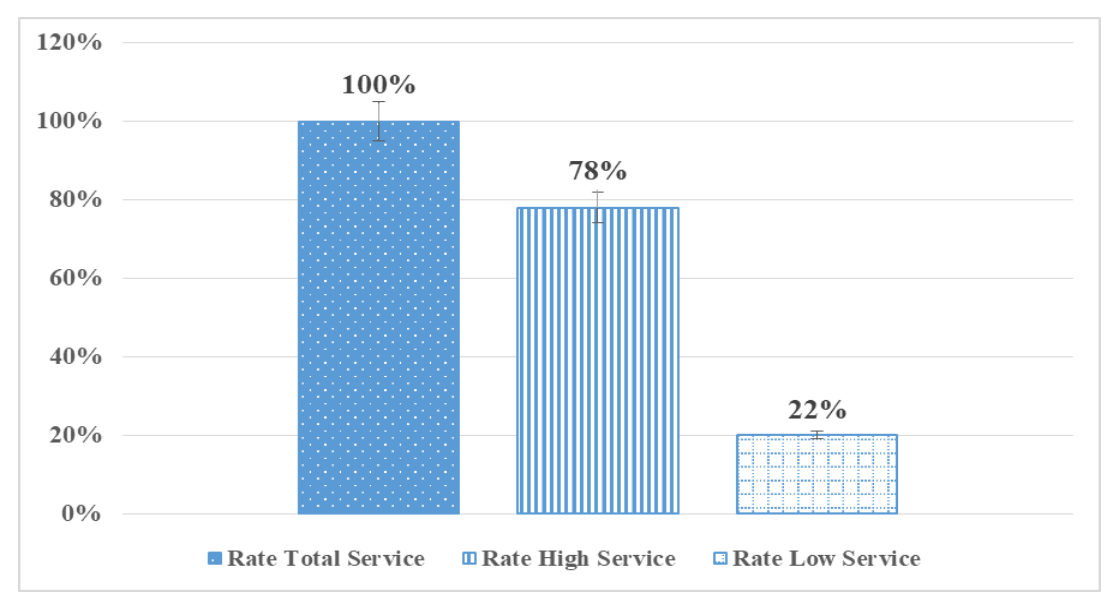

Figure 6. Percentage of low and stable services 
Table 10. Identification of weaker services and corresponding recommendations suggested

\begin{tabular}{|c|c|c|}
\hline QoS Criteria & QoS services weaker & Recommendations suggested \\
\hline $\begin{array}{l}\text { Smart homes } \\
\text { design interface }\end{array}$ & Design of data collection procedures $\left(\mathrm{QS}_{111}\right)$ & $\begin{array}{l}\text { Identify issues and/or opportunities } \\
\text { for collecting data }\end{array}$ \\
\hline Data acquisition & $\begin{array}{l}\text { Storage capacity. }\left(\mathrm{QS}_{426}\right) \\
\text { Critical data }\left(\mathrm{QS}_{427}\right)\end{array}$ & \\
\hline $\begin{array}{l}\text { Connection } \\
\text { technologies }\end{array}$ & Message scheduling $\left(\mathrm{QS}_{521}\right)$ & \\
\hline $\begin{array}{l}\text { Applications } \\
\text { technologies }\end{array}$ & Telemetry: technologies to exchange information $\left(\mathrm{QS}_{132}\right)$ & $\begin{array}{l}\text { Monotoring and tracking of things } \\
\text { automatically and replenished it. }\end{array}$ \\
\hline Remote control of & House Items $\left(\mathrm{QS}_{221}\right)$ & \\
\hline house system & $\begin{array}{l}\text { Virtual monitoring of important devices }\left(\mathrm{QS}_{223}\right) \\
\text { Remote Emergency }\left(\mathrm{QS}_{224}\right) \\
\text { Remote display and operation }\left(\mathrm{QS}_{225}\right)\end{array}$ & \\
\hline Human operator & $\begin{array}{l}\text { Human trust autonomous systems }\left(\mathrm{QS}_{311}\right) \\
\text { Monitoring for a house with Presence of people }\left(\mathrm{QS}_{312}\right)\end{array}$ & $\begin{array}{l}\text { Designing for human trust } \\
\text { autonomous systems }\end{array}$ \\
\hline $\begin{array}{l}\text { Decision-making } \\
\text { systems }\end{array}$ & Learning method $\left(\mathrm{QS}_{333}\right)$ & $\begin{array}{l}\text { The development of trusted operators } \\
\text { for mixed-use users could improve }\end{array}$ \\
\hline Sensor IoT & $\begin{array}{l}\text { Technology things appropriate sensor to collect data }\left(\mathrm{QS}_{411}\right) \\
\text { Power consumption rate }\left(\mathrm{QS}_{412}\right)\end{array}$ & $\begin{array}{l}\text { the combination of standalone } \\
\text { systems of the users. }\end{array}$ \\
\hline
\end{tabular}

\section{CONCLUSION}

This article highlighted limitations of evaluation for the sustainability quality of service. Sustainability quality of service is associated with complexity, and traditional assessment approaches are ineffective for dealing with such assessment. To compensate for these limitations, the FQSI model based on linguistic approximation and a fuzzy system for QoS was developed. This approach highlights the multipossibilities and ambiguities in measuring IoT system QoS. The concept model is proposed to be adopted for the smart home IoT system, through the initial development and implementation of the lifestyle concept. The FQSI is written in terms of ranges of values, gives decision-makers considerable flexibility in decisionmaking. Qualitatively, it gives an IoT system sustainability level of a smart home is the «Very sustainable quality of service» which promotes "extremely flexibility". As a perspective for future work, we endeavor to find solutions that use more methodologies that characterize QoS in IoT. In addition, we are also considering using efficient prediction methods for optimal QoS mechanisms in IoT systems, and more cooperative approaches to solving the measurement and evaluation problems in the QoS of IoT.

\section{REFERENCES}

[1] X. Jiang, H. Ding, H. Shi, and C. Li, "Novel QoS optimization paradigm for IoT systems with fuzzy logic and visual information mining integration," Neural Comput. Appl., vol. 32, no. 21, pp. 16427-16443, 2020, doi: 10.1007/s00521-019-04020-3.

[2] N. N. Srinidhi, S. M. Dilip Kumar, and K. R. Venugopal, "Network optimizations in the Internet of Things: A review," Eng. Sci. Technol. an Int. J., vol. 22, no. 1, pp. 1-21, 2019, doi: 10.1016/j.jestch.2018.09.003.

[3] W. M. Lafta, A. A. Alkadhmawee, and M. A. Altaha, "Best strategy to control data on internet-of-robotic-things in heterogeneous networks," Int. J. Electr. Comput. Eng., vol. 11, no. 2, pp. 1830-1838, 2021, doi: 10.11591/ijece.v11i2.pp1830-1838.

[4] S. Potluri and K. S. Rao, "Optimization model for QoS based task scheduling in cloud computing environment," Indones. J. Electr. Eng. Comput. Sci., vol. 18, no. 2, pp. 1081-1088, 2020, doi: 10.11591/ijeecs.v18.i2.pp1081-1088.

[5] F. Boumehrez, A. H. Sahour, and N. Doghmane, "Telehealth care enhancement using the internet of things technology," Bull. Electr. Eng. Informatics, vol. 10, no. 5, pp. 2652-2660, 2021, doi: 10.11591/eei.v10i5.2968.

[6] S. Wibowo and S. Grandhi, "Fuzzy multicriteria analysis for performance evaluation of internet-of-things-based supply chains," Symmetry (Basel)., vol. 10, no. 11, p. 603, 2018, doi: 10.3390/sym10110603.

[7] S. Wibowo, "A fuzzy approach for evaluating the performance of supply chain based on Internet of Things," in Proceedings 2012 IEEE International Conference on Cyber Technology in Automation, Control, and Intelligent Systems, CYBER 2012, 2012, pp. 275-280, doi: 10.1109/CYBER.2012.6392565.

[8] J. Li, L. Yang, X. Fu, F. Chao, and Y. Qu, "Dynamic QoS solution for enterprise networks using TSK fuzzy interpolation," in IEEE International Conference on Fuzzy Systems, 2017, pp. 1-6, doi: 10.1109/FUZZ-IEEE.2017.8015711.

[9] S. Kazmi, R. Liscano, and J. Ren, "Determining Fuzzy link quality membership functions in wireless sensor networks," Procedia Comput. Sci., vol. 34, pp. 149-156, 2014, doi: 10.1016/j.procs.2014.07.073.

[10] H. da S. Araújo et al., "A proposal for IoT dynamic routes selection based on contextual information," Sensors (Switzerland), vol. 18, no. 2, p. 353, 2018, doi: 10.3390/s18020353.

[11] S. Sankar and P. Srinivasan, "Fuzzy logic based energy aware routing protocol for internet of things," Int. J. Intell. Syst. Appl., vol. 10, no. 10, pp. 11-19, 2018, doi: 10.5815/ijisa.2018.10.02.

[12] F. Retima, S. Benharzallah, L. Kahloul, and O. Kazar, "A quality-aware context information selection based fuzzy logic in iot environment," Int. Arab J. Inf. Technol., vol. 15, no. 3A Special Issue, pp. 522-531, 2018.

[13] Y. Akbari and S. Tabatabaei, "A New Method to Find a High Reliable Route in IoT by Using Reinforcement Learning and Fuzzy Logic," Wirel. Pers. Commun., vol. 112, no. 2, pp. 967-983, 2020, doi: 10.1007/s11277-020-07086-8.

[14] J. Shang, S. Li, and J. Huang, "A robust fuzzy local Information c-means clustering algorithm with noise detection," in Ninth International Conference on Graphic and Image Processing (ICGIP 2017), 2018, vol. 10615, p. $106151 \mathrm{Z}$.

[15] A. Segatori, A. Bechini, P. Ducange, and F. Marcelloni, "A Distributed Fuzzy Associative Classifier for Big Data," IEEE Trans. Cybern., vol. 48, no. 9, pp. 2656-2669, 2018, doi: 10.1109/TCYB.2017.2748225. 
[16] C. T. Lin, H. Chiu, and Y. H. Tseng, “Agility evaluation using fuzzy logic,” Int. J. Prod. Econ., vol. 101, no. 2, pp. 353-368, 2006, doi: 10.1016/j.ijpe.2005.01.011.

[17] S. Rajak and S. Vinodh, "Application of fuzzy logic for social sustainability performance evaluation: a case study of an Indian automotive component manufacturing organization," J. Clean. Prod., vol. 108, pp. 1184-1192, 2015.

[18] S. Rajak, P. Parthiban, and R. Dhanalakshmi, "Sustainable transportation systems performance evaluation using fuzzy logic," Ecol. Indic., vol. 71, pp. 503-513, 2016, doi: 10.1016/j.ecolind.2016.07.031.

[19] S. Hendiani, H. Liao, M. Bagherpour, M. Tvaronavičienè, A. Banaitis, and J. Antucheviciene, "Analyzing the status of sustainable development in the manufacturing sector using multi-expert multi-criteria fuzzy decision-making and integrated triple bottom lines," Int. J. Environ. Res. Public Health, vol. 17, no. 11, p. 3800, 2020, doi: 10.3390/ijerph17113800.

[20] T. J. Ross, "Fuzzy Logic with Engineering Applications: Third Edition," Fuzzy Log. with Eng. Appl. Third Ed., vol. 25, no. 4, p. 71, 2010, doi: 10.1002/9781119994374.

[21] Mathworks.com, 'Mathworks. MathWorks-Editeur de MATLAB et Simulink-MATLAB \& Simulink', 2021. [Online]. Available: https://www.mathworks.com/products/new_products/release2019b.html. [Accessed: 12-May-2019].

[22] H. G. Hamid and Z. T. Alisa, "Survey on IoT application layer protocols," Indones. J. Electr. Eng. Comput. Sci., vol. 21, no. 3, pp. 1663-1672, 2021, doi: 10.11591/ijeecs.v21.i3.pp1663-1672.

[23] A. A. Abbood, Q. M. Shallal, and M. A. Fadhel, "Internet of things (IoT): A technology review, security issues, threats, and open challenges," Indones. J. Electr. Eng. Comput. Sci., vol. 20, no. 3, pp. 1685-1692, 2020, doi: 10.11591/ijeecs.v20.i3.pp1685-1692.

[24] M. Murad, O. Bayat, and H. M. Marhoon, "Design and implementation of a smart home system with two levels of security based on IoT technology," Indones. J. Electr. Eng. Comput. Sci., vol. 21, no. 1, pp. 546-557, 2021.

[25] H. M. Hoe and M. P. Abdullah, "Smart home appliances scheduling considering user comfort level," Indones. J. Electr. Eng. Comput. Sci., vol. 20, no. 2, pp. 593-601, 2020, doi: 10.11591/ijeecs.v20.i2.pp593-601.

[26] M. A. Omran, W. K. Saad, B. J. Hamza, and A. Fahe, "A survey of various intelligent home applications using IoT and intelligent controllers,” Indones. J. Electr. Eng. Comput. Sci., vol. 23, no. 1, pp. 490-499, 2021, doi: 10.11591/ijeecs.v23.i1.pp490-499.

[27] P. Sethi and S. R. Sarangi, "Internet of Things: Architectures, Protocols, and Applications," J. Electr. Comput. Eng., vol. 2017, pp. 1-147, 2017, doi: 10.1155/2017/9324035.

[28] H. Qi, "Fuzzy logic hybridized artificial intelligence for computing and networking on internet of things platform," Peer-to-Peer Netw. Appl., vol. 13, no. 6, pp. 2078-2088, 2020, doi: 10.1007/s12083-019-00827-y.

[29] A. A. Abbood, Q. M. Shallal, and M. A. Fadhel, "Internet of things (IoT): A technology review, security issues, threats, and open challenges," Indones. J. Electr. Eng. Comput. Sci., vol. 20, no. 3, pp. 1685-1692, 2020, doi: 10.11591/ijeecs.v20.i3.pp1685-1692.

[30] H. M. Hoe and M. P. Abdullah, "Smart home appliances scheduling considering user comfort level," Indones. J. Electr. Eng. Comput. Sci., vol. 20, no. 2, pp. 593-601, 2020, doi: 10.11591/ijeecs.v20.i2.pp593-601.

[31] L. S. Abdulla, M. K. Mahmood, A. F. Salih, and S. M. Karim, "Analysis and evaluation of symmetric key ciphers for internet of things smart home," Indones. J. Electr. Eng. Comput. Sci., vol. 22, no. 2, pp. 1191-1198, 2021, doi: 10.11591/IJEECS.V22.I2.PP1191-1198.

\section{BIOGRAPHIES OF AUTHORS}
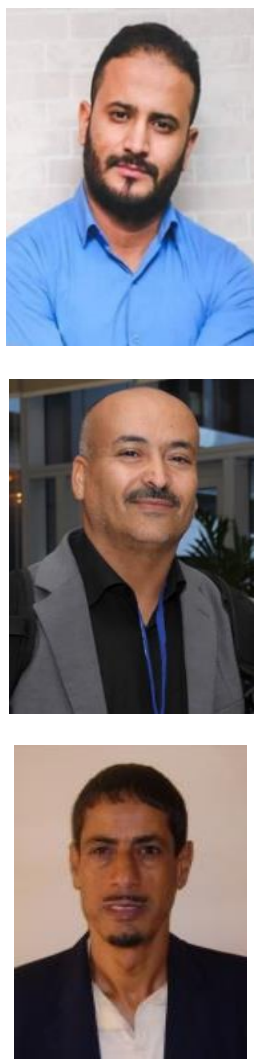

Lairedj Aboubaker Saddik (D) SC P He obtained a Bachelor's degree in Engineering in Computer Science from Tahiri University Mohamed Bechar (Algeria) in June 2012 and a PhD student from Bechar University (Algeria) since 2016. The title of his doctoral is "Mechanism of Quality of services for Internet of things" and the main objective was to build an effective mechanism to determine the efficiency of services for the operating systems Under the internet of things. His research interests include Wireless Sensor Networks, Internet of Things. Quality of Service. Wireless sensor network coverage and connection, feature extraction. Participated in many national and international conferences. $\mathrm{He}$ can be contacted at: Lairedjboubakerdz@gmail.com.

Prof. Benahmed khelifa (D) 8 SC P has done his research for Ph.D degree in the field of wireless sensor networks at the school of computing and mathematical sciences at Liverpool john moores university, UK. He has 30 years of experience in education and research at the University Tahri Mohamed Bechar, Algeria. Khelifa has also several international publications in the fields: wireless sensor networks, security, surveillance, smart grid, Internet of things, Smart agriculture, Smart transport. IndexH(10), IndexI(10), Citations(328). Khelifa Benahmed holds Engineer, Magister degrees and $\mathrm{PhD}$ in Computer sciences in University of Oran Algeria. He can be contacted at email: benahmed_khelifa@yahoo.fr.

Bounaama Fatah (iD $8 \mathrm{SC}$ P received the Electronics Engineering degree from the University of Sciences and Technology of Oran (Algeria) in June 1988 and a MEng and PhD from the University of Bechar (Algeria) in 2001 and 2010 respectively. His scientific interests are Simulation, Identification, Control and thier Implementation on embeded system applications in agriculture and horticulture environment of greenhouse. He can be contacted at email: fbounaama2002@yahoo.fr. 